Supporting Information

\title{
The Absolute Configuration of trans-Perhydroazulene
}

Fumito Saito, ${ }^{1}$ Dennis Gerbig, ${ }^{1}$ Jonathan Becker, ${ }^{2}$ and Peter R. Schreinerl *

${ }^{1}$ Institute of Organic Chemistry, Justus Liebig University

Heinrich-Buff-Ring 17, 35392 Giessen, Germany

${ }^{2}$ Institute of Inorganic and Analytical Chemistry, Justus Liebig University

Heinrich-Buff-Ring 17, 35392 Giessen, Germany

prs@uni-giessen.de

\section{Table of Contents}

Synthesis and Characterization of New Compounds......................................................... 2

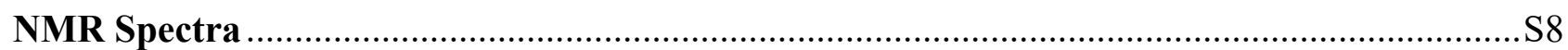

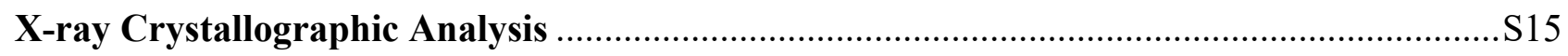

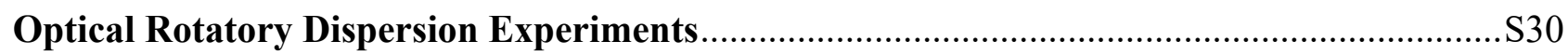

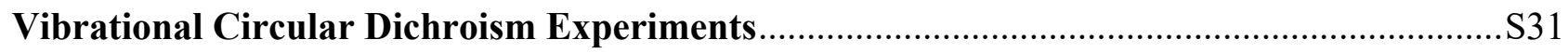

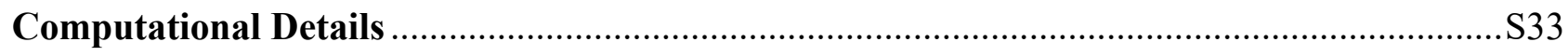

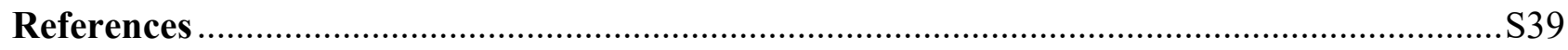




\section{Synthesis and Characterization of New Compounds}

\section{General Experimental Methods}

All air- and water sensitive reactions were carried out under $\mathrm{N}_{2}$ atmosphere using standard Schlenk techniques. Reactions that required heating were carried out using an oil bath. All chemicals and common organic solvents (ACS or HPLC grade) were used as supplied. Thin layer chromatography (TLC) was performed plastic-backed silica gel 60 coated with a fluorescence indicator and visualized by UV quenching and/or staining with phosphomolybdic acid (5\% in EtOH) or $\mathrm{KMnO}_{4}(0.75 \mathrm{~g}$ $\mathrm{KMnO}_{4}, 5 \mathrm{~g} \mathrm{~K}_{2} \mathrm{CO}_{3}, 70 \mathrm{mg} \mathrm{NaOH}, 100 \mathrm{~mL} \mathrm{H}{ }_{2} \mathrm{O}$ ) followed by warming with a heat gun. Flash column chromatography was performed under a forced-flow of $\mathrm{N}_{2}$ using Merck silica gel $60(0.040-0.063$ $\mathrm{mm}$ particle size). NMR spectra were recorded on a Bruker spectrometer Avance II $400 \mathrm{MHz}$ (AV 400). Chemical shifts $(\delta)$ are given in ppm relative to residual solvent peaks. Data for ${ }^{1} \mathrm{H}$ NMR are reported as follows: chemical shift (multiplicity, coupling constants where applicable, number of hydrogens). IR spectra were recorded on an ALPHA spectrometer from a Bruker Optics and only peaks diagnostic for major functional groups are reported in frequency of absorption $\left(\mathrm{cm}^{-1}\right)$. Highresolution mass spectra (HRMS) were measured on a Bruker microTOF spectrometer, and the mass for the found ions are given in $m / z$ units. Optical rotations were measured on a polarimeter P-2000 from JASCO at the sodium D line with a $50 \mathrm{~mm}$ optical path length and reported as $[\alpha]_{\mathrm{D}}{ }^{25}$ (concentration (g/100 mL), solvent). Melting points (m.p.) were measured on a Krüss Optronic melting point meter KSP1N and are reported uncorrected.

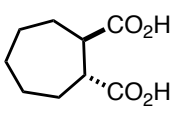

$( \pm)-4$

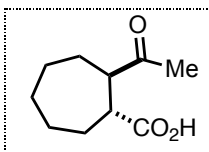

S1

rac-Dicarboxylic acid ( \pm -4 $\mathbf{4}$ was prepared following a modified literature procedure: ${ }^{1}$ To a solution of $\mathbf{S 1}^{1}$ (6.0 g, $33 \mathrm{mmol}, 1.0$ equiv) in aq $\mathrm{NaOCl}(130 \mathrm{~mL}, 10-15 \%$ active chlorine) was added a solution of $\mathrm{NaOH}\left(2.0 \mathrm{~g}, 50 \mathrm{mmol}, 1.5\right.$ equiv) in $\mathrm{H}_{2} \mathrm{O}(15 \mathrm{~mL})$. Within a few minutes, an exothermic reaction was observed together with precipitation of a white solid. Once the reaction had cooled to room temperature, the solution was stirred for further $30 \mathrm{~min}$. The mixture was cooled to $0{ }^{\circ} \mathrm{C}$ and excess $\mathrm{NaOCl}$ was quenched with $\mathrm{Na}_{2} \mathrm{~S}_{2} \mathrm{O}_{5}(3.7 \mathrm{~g})$ in $\mathrm{H}_{2} \mathrm{O}$. After acidification with aq $\mathrm{H}_{2} \mathrm{SO}_{4}$ to $\mathrm{pH}$ 1, the mixture was extracted with $\mathrm{Et}_{2} \mathrm{O}(5 \mathrm{x})$ and the combined extracts were dried over $\mathrm{Na}_{2} \mathrm{SO}_{4}$, filtered, and concentrated under reduced pressure to give $( \pm)-4(6.0 \mathrm{~g}, 98 \%)$ as a white solid: m.p: 142-143 ${ }^{\circ} \mathrm{C}$; IR (KBr) 1696, 1293, $950 \mathrm{~cm}^{-1} ;{ }^{1} \mathrm{H} \mathrm{NMR}\left(\mathrm{CDCl}_{3}, 400 \mathrm{MHz}\right) \delta 10.35$ (br s, 2H), $2.87-$ 
$2.76(\mathrm{~m}, 2 \mathrm{H}), 2.12-2.00(\mathrm{~m}, 2 \mathrm{H}), 1.84-1.51(\mathrm{~m}, 8 \mathrm{H}) ;{ }^{13} \mathrm{C}\left\{{ }^{1} \mathrm{H}\right\} \mathrm{NMR}\left(\mathrm{CDCl}_{3}, 101 \mathrm{MHz}\right) \delta 182.7$, 47.3, 30.2, 27.7, 26.3; HRMS (ESI) calcd for $\mathrm{C}_{9} \mathrm{H}_{14} \mathrm{NaO}_{4}[\mathrm{M}+\mathrm{Na}]^{+}:$209.0784, found: 209.0784 .

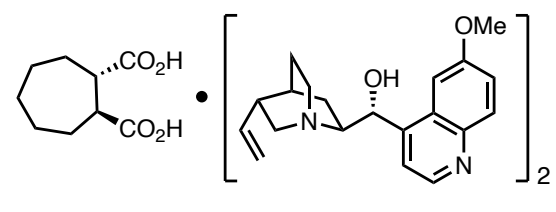

$(-)-5$

The resolution of $( \pm)-4$ was performed following a modified literature procedure: ${ }^{2}$ Quinine $(47.4 \mathrm{~g}$, $146 \mathrm{mmol}, 2.0$ equiv) was dissolved at $80{ }^{\circ} \mathrm{C}$ in a mixture of EtOAc $(265 \mathrm{~mL})$ and EtOH $(30 \mathrm{~mL})$. To this was added a solution of $( \pm)-4$ (13.6 g, $73 \mathrm{mmol}, 1.0$ equiv) in EtOH (40 mL). The resulting solution was allowed to stand at room temperature overnight. The crystals formed were collected by filtration and recrystallized five times from 50\% aq EtOH to give (-)-5 (12.3 g, 20\%) as colorless crystals: m.p. $142-143^{\circ} \mathrm{C} ;[\alpha]_{\mathrm{D}}{ }^{25}=-140.1(\mathrm{c} 1.65, \mathrm{EtOH})$.

\section{Determination of enantiopurity of the resolved acid (-)-4.}

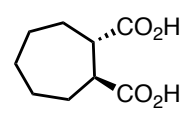

$(-)-4$

$(-)-5(0.79 \mathrm{~g} 0.95 \mathrm{mmol})$ was suspended in $\mathrm{Et}_{2} \mathrm{O}(15 \mathrm{~mL})$. To this was added $1 \mathrm{M}$ aq $\mathrm{HCl}(15 \mathrm{~mL})$ at $0{ }^{\circ} \mathrm{C}$ and the biphasic mixture was stirred at $0{ }^{\circ} \mathrm{C}$ for $5 \mathrm{~min}$. The two phases were separated, and the aqueous phase was extracted with $\mathrm{Et}_{2} \mathrm{O}(4 \mathrm{x})$. The combined organic phases were dried over $\mathrm{Na}_{2} \mathrm{SO}_{4}$, filtered and concentrated under reduced pressure to give (-)-4 (0.18 g, quant) as a white solid: m.p. $110-111^{\circ} \mathrm{C} ;[\alpha]_{\mathrm{D}}^{25}=-28.2(\mathrm{c} 1.38, \mathrm{EtOH})$.

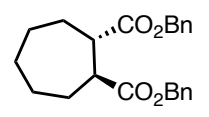

$(+)-\mathbf{S 2}$

To a solution of (-)-4 (47 mg, $0.25 \mathrm{mmol}, 1.0$ equiv) in $\mathrm{CH}_{2} \mathrm{Cl}_{2}(1.3 \mathrm{~mL})$ were added DIC (86 $\mu \mathrm{L}$, $0.55 \mathrm{mmol}, 2.2$ equiv), benzyl alcohol $(57 \mu \mathrm{L}, 0.55 \mathrm{mmol}, 2.2$ equiv), followed by catalytic amounts of DMAP. The mixture was stirred at room temperature for $2.5 \mathrm{~h}$, directly loaded onto a silica gel column and purified by flash column chromatography (pentane/Et $2 \mathrm{O} 9: 1 \rightarrow 4: 1$ ) to give $(+)-\mathbf{S 2}$ $(72 \mathrm{mg}, 79 \%)$ as a clear colorless oil: IR (thin film) $1729,1250,1156 \mathrm{~cm}^{-1} ;[\alpha]_{\mathrm{D}}{ }^{25}=+2.9(\mathrm{c} 1.2$, 
$\left.\mathrm{CHCl}_{3}\right) ;{ }^{1} \mathrm{H} \mathrm{NMR}\left(\mathrm{CDCl}_{3}, 400 \mathrm{MHz}\right) \delta 7.37-7.27(\mathrm{~m}, 10 \mathrm{H}), 5.10-4.98(\mathrm{~m}, 4 \mathrm{H}), 3.04-2.96(\mathrm{~m}$, 2H), $2.02-1.92(\mathrm{~m}, 2 \mathrm{H}), 1.80-1.61(\mathrm{~m}, 4 \mathrm{H}), 1.60-1.49(\mathrm{~m}, 4 \mathrm{H}) ;{ }^{13} \mathrm{C}\left\{{ }^{1} \mathrm{H}\right\} \mathrm{NMR}\left(\mathrm{CDCl}_{3}, 101 \mathrm{MHz}\right)$ $\delta$ 175.5, 136.0, 128.5, 128.1, 66.3, 47.0, 29.7, 28.6, 26.4; HRMS (ESI) calcd for $\mathrm{C}_{23} \mathrm{H}_{26} \mathrm{NaO}_{4}$ $[\mathrm{M}+\mathrm{Na}]^{+}:$389.1723, found: 389.1723; ee = 99\% (NP-HPLC, column: Chiralpak IB; eluent: $n$ hexane/ $i \mathrm{PrOH}$ 98:2; flow rate: $1.2 \mathrm{~mL} / \mathrm{min}$; detection: $254 \mathrm{~nm}$, retention time: $5.5 \mathrm{~min}$ (minor), $5.8 \min$ (major)).
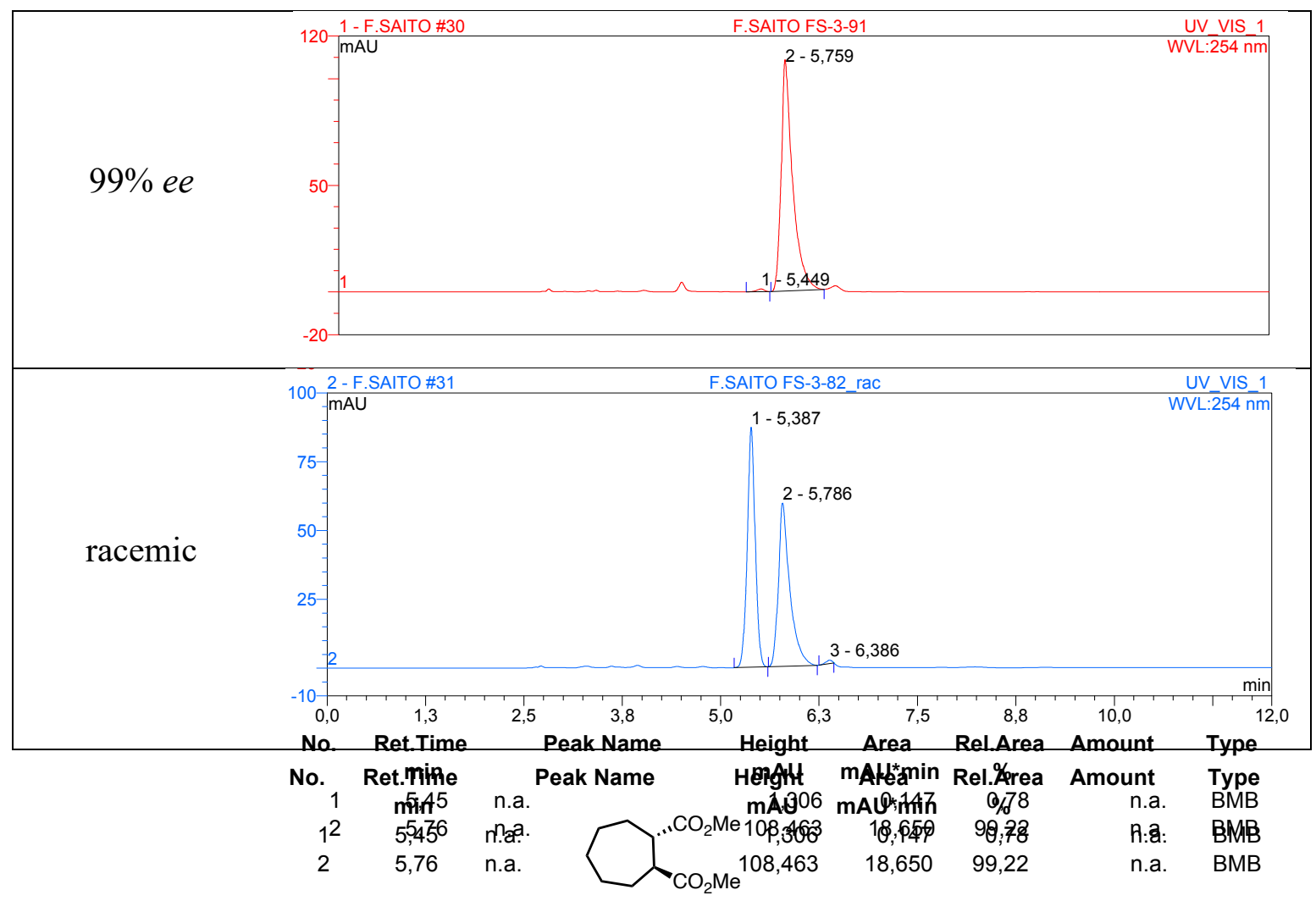

$(-)-6$

(-)-5 (11.3 g, $13.5 \mathrm{mmol})$ was suspended in $\mathrm{Et}_{2} \mathrm{O}(200 \mathrm{~mL})$. To this was added $1 \mathrm{M}$ aq $\mathrm{HCl}(200 \mathrm{~mL})$ at $0{ }^{\circ} \mathrm{C}$ and the biphasic mixture was stirred at $0{ }^{\circ} \mathrm{C}$ for $2 \mathrm{~min}$. The two phases were separated, and the aqueous phase was extracted with $\mathrm{Et}_{2} \mathrm{O}(4 \mathrm{x})$. The combined organic phases were dried over $\mathrm{Na}_{2} \mathrm{SO}_{4}$, filtered and concentrated under reduced pressure to give (-)-4 (2.41 g, 96\%) as a white solid. To a solution of (-)-4 (2.2 g, $12 \mathrm{mmol})$ in $\mathrm{MeOH}(30 \mathrm{~mL})$ was added conc $\mathrm{H}_{2} \mathrm{SO}_{4}(1.2 \mathrm{~mL})$ dropwise at $0{ }^{\circ} \mathrm{C}$ and the mixture was stirred at $40{ }^{\circ} \mathrm{C}$ for $20 \mathrm{~h} . \mathrm{MeOH}$ was evaporated under reduced pressure before the residue was diluted with $\mathrm{H}_{2} \mathrm{O}$ and extracted with $\mathrm{Et}_{2} \mathrm{O}(3 \mathrm{x})$. The combined organic phases were washed with brine, dried over $\mathrm{Na}_{2} \mathrm{SO}_{4}$, filtered, and concentrated under reduced pressure to give (-)-6 (2.3 g, 85\% for 2 steps) as a pale yellow oil: IR (thin film) $1734,1199,1166 \mathrm{~cm}^{-1} ;[\alpha]_{\mathrm{D}}{ }^{25}=-$ $19.6\left(\mathrm{c} 1.3, \mathrm{CHCl}_{3}\right) ;{ }^{1} \mathrm{H} \mathrm{NMR}\left(\mathrm{CDCl}_{3}, 400 \mathrm{MHz}\right) \delta 3.66(\mathrm{~s}, 6 \mathrm{H}), 2.96-2.89(\mathrm{~m}, 2 \mathrm{H}), 1.98$ - $1.89(\mathrm{~m}$, 
2H), $1.77-1.64(\mathrm{~m}, 4 \mathrm{H}), 1.59-1.50(\mathrm{~m}, 4 \mathrm{H}) ;{ }^{13} \mathrm{C}\left\{{ }^{1} \mathrm{H}\right\} \mathrm{NMR}\left(\mathrm{CDCl}_{3}, 101 \mathrm{MHz}\right) \delta 176.3,51.8,46.8$, 29.7, 28.7, 26.4; HRMS (ESI) calcd for $\mathrm{C}_{11} \mathrm{H}_{18} \mathrm{NaO}_{4}[\mathrm{M}+\mathrm{Na}]^{+}$: 237.1097, found: 237.1099.

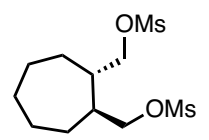

$(+)-7$

$\mathrm{LiAlH}_{4}\left(1.3 \mathrm{~g}, 34 \mathrm{mmol}, 3.0\right.$ equiv) was suspended in THF $(100 \mathrm{~mL})$ at $0{ }^{\circ} \mathrm{C}$. To this was slowly added a solution of (-)-6 (2.4 g, $11 \mathrm{mmol}, 1.0$ equiv $)$ in THF $(10 \mathrm{~mL})$ at $0{ }^{\circ} \mathrm{C}$. The mixture was stirred at room temperature for $2 \mathrm{~h}$. The reaction was quenched at $0{ }^{\circ} \mathrm{C}$ by sequential addition of $\mathrm{H}_{2} \mathrm{O}$ $(1.3 \mathrm{~mL}), 15 \%$ aq $\mathrm{NaOH}(1.3 \mathrm{~mL})$, and $\mathrm{H}_{2} \mathrm{O}(3.9 \mathrm{~mL})$. The mixture was filtered through Celite (rinsed with THF) and the filtrate was concentrated under reduced pressure to give a crude product $(2.3 \mathrm{~g}$, quant).

To a solution of the crude diol (2.3 g, $11 \mathrm{mmol}, 1.0$ equiv) and $\mathrm{NEt}_{3}$ (4.7 mL, $34 \mathrm{mmol}, 3.0$ equiv) in $\mathrm{CH}_{2} \mathrm{Cl}_{2}(50 \mathrm{~mL})$ was dropwise added a solution of methanesulfonyl chloride $(2.1 \mathrm{~mL}, 27 \mathrm{mmol}$, 2.4 equiv $)$ in $\mathrm{CH}_{2} \mathrm{Cl}_{2}(10 \mathrm{~mL})$ via a dropping funnel. After complete addition, the mixture was stirred at room temperature for $2.5 \mathrm{~h} . \mathrm{H}_{2} \mathrm{O}$ was added and the two phases were separated. The aqueous phase was extracted with $\mathrm{CH}_{2} \mathrm{Cl}_{2}(2 \mathrm{x})$. The combined organic phases were washed with brine, dried over $\mathrm{Na}_{2} \mathrm{SO}_{4}$, filtered, and concentrated under reduced pressure. The residue was purified by flash column chromatography $\left(\mathrm{CH}_{2} \mathrm{Cl}_{2} / \mathrm{MeOH} 40: 1 \rightarrow 30: 1\right)$ to give $(+)-7$ (3.5 g, quant) as a pale orange solid: m.p. 67-69 ${ }^{\circ} \mathrm{C}$; IR (KBr) 1351, $1174 \mathrm{~cm}^{-1} ;[\alpha]_{\mathrm{D}}{ }^{25}=+13.2\left(\mathrm{c} 1.25, \mathrm{CHCl}_{3}\right) ;{ }^{1} \mathrm{H} \mathrm{NMR}\left(\mathrm{CDCl}_{3}, 400 \mathrm{MHz}\right)$ $\delta 4.22$ - $4.12(\mathrm{~m}, 4 \mathrm{H}), 3.03(\mathrm{~s}, 6 \mathrm{H}), 1.87$ - $1.79(\mathrm{~m}, 2 \mathrm{H}), 1.77$ - $1.59(\mathrm{~m}, 6 \mathrm{H}), 1.57$ - $1.40(\mathrm{~m}, 4 \mathrm{H})$; ${ }^{13} \mathrm{C}\left\{{ }^{1} \mathrm{H}\right\} \mathrm{NMR}\left(\mathrm{CDCl}_{3}, 101 \mathrm{MHz}\right) \delta 72.8,39.7,37.4,30.0,28.9,26.2$; HRMS (ESI) calcd for $\mathrm{C}_{11} \mathrm{H}_{22} \mathrm{NaO}_{6} \mathrm{~S}_{2}[\mathrm{M}+\mathrm{Na}]^{+}: 337.0750$, found: 337.0748 .

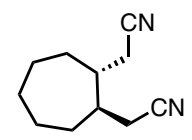

$(+)-8$

To a solution of (+)-7 (3.4 g, $11 \mathrm{mmol}, 1.0$ equiv) in DMSO (100 mL) was added KCN (2.1 g, $32 \mathrm{mmol}, 3.0$ equiv) and the mixture was stirred at $95{ }^{\circ} \mathrm{C}$ for $15 \mathrm{~h}$. After cooling to room temperature, $\mathrm{H}_{2} \mathrm{O}$ was added, and the mixture was extracted with $\mathrm{Et}_{2} \mathrm{O}(3 \mathrm{x})$. The combined organic phases were washed with brine, dried over $\mathrm{Na}_{2} \mathrm{SO}_{4}$, filtered, and concentrated under reduced pressure. The residue was purified by flash column chromatography (pentane/ $\left.\mathrm{Et}_{2} \mathrm{O} 1: 1\right)$ to give $(+)-8(1.4 \mathrm{~g}, 74 \%)$ as a pale yellow oil: IR (thin film) $2245 \mathrm{~cm}^{-1} ;[\alpha]_{\mathrm{D}}{ }^{25}=+27.5\left(\mathrm{c} 0.88, \mathrm{CHCl}_{3}\right) ;{ }^{1} \mathrm{H} \mathrm{NMR}\left(\mathrm{CDCl}_{3}, 400 \mathrm{MHz}\right) \delta$ 
$2.48-2.36(\mathrm{~m}, 4 \mathrm{H}), 1.85-1.64(\mathrm{~m}, 8 \mathrm{H}), 1.61-1.45(\mathrm{~m}, 4 \mathrm{H}) ;{ }^{13} \mathrm{C}\left\{{ }^{1} \mathrm{H}\right\} \mathrm{NMR}\left(\mathrm{CDCl}_{3}, 101 \mathrm{MHz}\right) \delta$ 118.3, 40.0, 31.8, 28.6, 25.4, 23.8; HRMS (ESI) calcd for $\mathrm{C}_{11} \mathrm{H}_{16} \mathrm{~N}_{2} \mathrm{Na}[\mathrm{M}+\mathrm{Na}]^{+}:$199.1205, found: 199.1204.

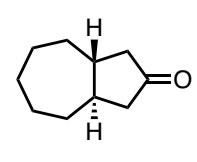

$(-)-9$

To a solution of $(+)-8(1.3 \mathrm{~g}, 7.1 \mathrm{mmol})$ in THF $(20 \mathrm{~mL})$ was added $\mathrm{NaH}(60 \%$ in mineral oil, $0.43 \mathrm{~g}$, $11 \mathrm{mmol}, 1.5$ equiv) at room temperature. The mixture was stirred at reflux for $2.5 \mathrm{~h}$. After cooling to room temperature, the solution was quenched by $\mathrm{H}_{2} \mathrm{O}$ and extracted with $\mathrm{Et}_{2} \mathrm{O}(3 \mathrm{x})$. The combined organic extracts were washed brine, dried over $\mathrm{Na}_{2} \mathrm{SO}_{4}$, filtered, and concentrated under reduced pressure to give a crude product as a yellow-orange solid.

The crude imino nitrile was dissolved in $\mathrm{EtOH}(15 \mathrm{~mL})$ and $6 \mathrm{M}$ aq $\mathrm{HCl}$ was added. The mixture was stirred at room temperature for $1 \mathrm{~h}$, diluted with $\mathrm{H}_{2} \mathrm{O}$, and extracted with $\mathrm{Et}_{2} \mathrm{O}(3 \mathrm{x})$. The combined organic extracts were washed brine, dried over $\mathrm{Na}_{2} \mathrm{SO}_{4}$, filtered, and concentrated under reduced pressure to give a crude product as a pale yellow solid.

The crude $\alpha$-cyano ketone was suspended in $33 \%$ aq $\mathrm{H}_{2} \mathrm{SO}_{4}(40 \mathrm{~mL})$ and the mixture was refluxed for $4 \mathrm{~h}$. After cooling to room temperature, the mixture was extracted with $\mathrm{Et}_{2} \mathrm{O}(3 \mathrm{x})$. The combined organic extracts were dried over $\mathrm{Na}_{2} \mathrm{SO}_{4}$, filtered and concentrated under reduced pressure. The residue was purified by flash column chromatography (pentane/ $\left.\mathrm{Et}_{2} \mathrm{O} 4: 1\right)$ to give (-)-9 (0.92 g, 85\% for 3 steps) as a pale yellow oil: IR (thin film) $1745 \mathrm{~cm}^{-1} ;[\alpha]_{\mathrm{D}}{ }^{25}=-185.6\left(\mathrm{c} 1.14, \mathrm{CHCl}_{3}\right) ;{ }^{1} \mathrm{H} \mathrm{NMR}$ $\left(\mathrm{CDCl}_{3}, 400 \mathrm{MHz}\right) \delta 2.52-2.38(\mathrm{~m}, 2 \mathrm{H}), 2.07-1.96(\mathrm{~m}, 2 \mathrm{H}), 1.95-1.82(\mathrm{~m}, 4 \mathrm{H}), 1.78-1.65(\mathrm{~m}$, 2H), $1.64-1.50(\mathrm{~m}, 4 \mathrm{H}), 1.34-1.22(\mathrm{~m}, 2 \mathrm{H}) ;{ }^{13} \mathrm{C}\left\{{ }^{1} \mathrm{H}\right\} \mathrm{NMR}\left(\mathrm{CDCl}_{3}, 101 \mathrm{MHz}\right) \delta 219.0,48.0,43.4$, 34.5, 27.1, 26.7; HRMS (ESI) calcd for $\mathrm{C}_{10} \mathrm{H}_{16} \mathrm{NaO}[\mathrm{M}+\mathrm{Na}]^{+}:$175.1092, found: 175.1095 .

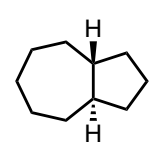

$(+)-1$

To a solution of (-)-9 (0.60 g, $3.9 \mathrm{mmol}, 1.0$ equiv) in $\mathrm{MeOH}(2.6 \mathrm{~mL})$ was added $p$-toluenesulfonyl hydrazide ( $0.81 \mathrm{~g}, 4.3 \mathrm{mmol}, 1.1$ equiv), and the mixture was stirred at room temperature. Within 2$3 \mathrm{~min}$, white precipitates started to appear and $\mathrm{MeOH}(1.0 \mathrm{~mL})$ was added to the mixture. After stirring for $40 \mathrm{~min}$, the mixture was filtered and the white solid was washed with $\mathrm{MeOH}$ and dried $(1.2 \mathrm{~g}, 93 \%)$. 
The crude tosylhydrazone ( $1.1 \mathrm{~g}, 3.4 \mathrm{mmol}, 1.0$ equiv) was dissolved in sulfolane/DMF (16 mL, 1:1), and to this were added $p-\mathrm{TsOH} \cdot \mathrm{H}_{2} \mathrm{O}(130 \mathrm{mg})$, followed by $\mathrm{NaBH}_{3} \mathrm{CN}(0.86 \mathrm{~g}, 14 \mathrm{mmol}, 4.0$ equiv $)$. The mixture was stirred at $100{ }^{\circ} \mathrm{C}$ for $16 \mathrm{~h}$ and allowed to cool to room temperature. The reaction mixture was partitioned between pentane and $\mathrm{H}_{2} \mathrm{O}$. The aqueous phase was extracted with pentane $(2 \mathrm{x})$, and the combined extracts were washed with brine, dried over $\mathrm{Na}_{2} \mathrm{SO}_{4}$, filtered and concentrated under reduced pressure. The residue was purified by flash column chromatography (pentane) to give $(+)-1(0.18 \mathrm{~g}, 35 \%$ for 2 steps $)$ as a clear colorless liquid: $[\alpha]_{\mathrm{D}}{ }^{25}=+13.1\left(\mathrm{c} 0.525, \mathrm{CDCl}_{3}\right) ;{ }^{1} \mathrm{H} \mathrm{NMR}$ $\left(\mathrm{CDCl}_{3}, 400 \mathrm{MHz}\right) \delta 1.92-1.76(\mathrm{~m}, 4 \mathrm{H}), 1.70-1.58(\mathrm{~m}, 2 \mathrm{H}), 1.57-1.41(\mathrm{~m}, 8 \mathrm{H}), 1.24-1.09(\mathrm{~m}$, $4 \mathrm{H}) ;{ }^{13} \mathrm{C}\left\{{ }^{1} \mathrm{H}\right\} \mathrm{NMR}\left(\mathrm{CDCl}_{3}, 101 \mathrm{MHz}\right) \delta 46.4,35.2,34.3,28.0,26.6,24.0$. NMR spectral data matched with those reported previously. ${ }^{3}$ 


\section{NMR Spectra}

rac-Dicarboxylic acid $( \pm)-4$

${ }^{1} \mathrm{H}$ NMR (400 MHz, $\left.\mathrm{CDCl}_{3}\right)$<smiles>O=C(O)C1CCCCC1C(=O)O</smiles>
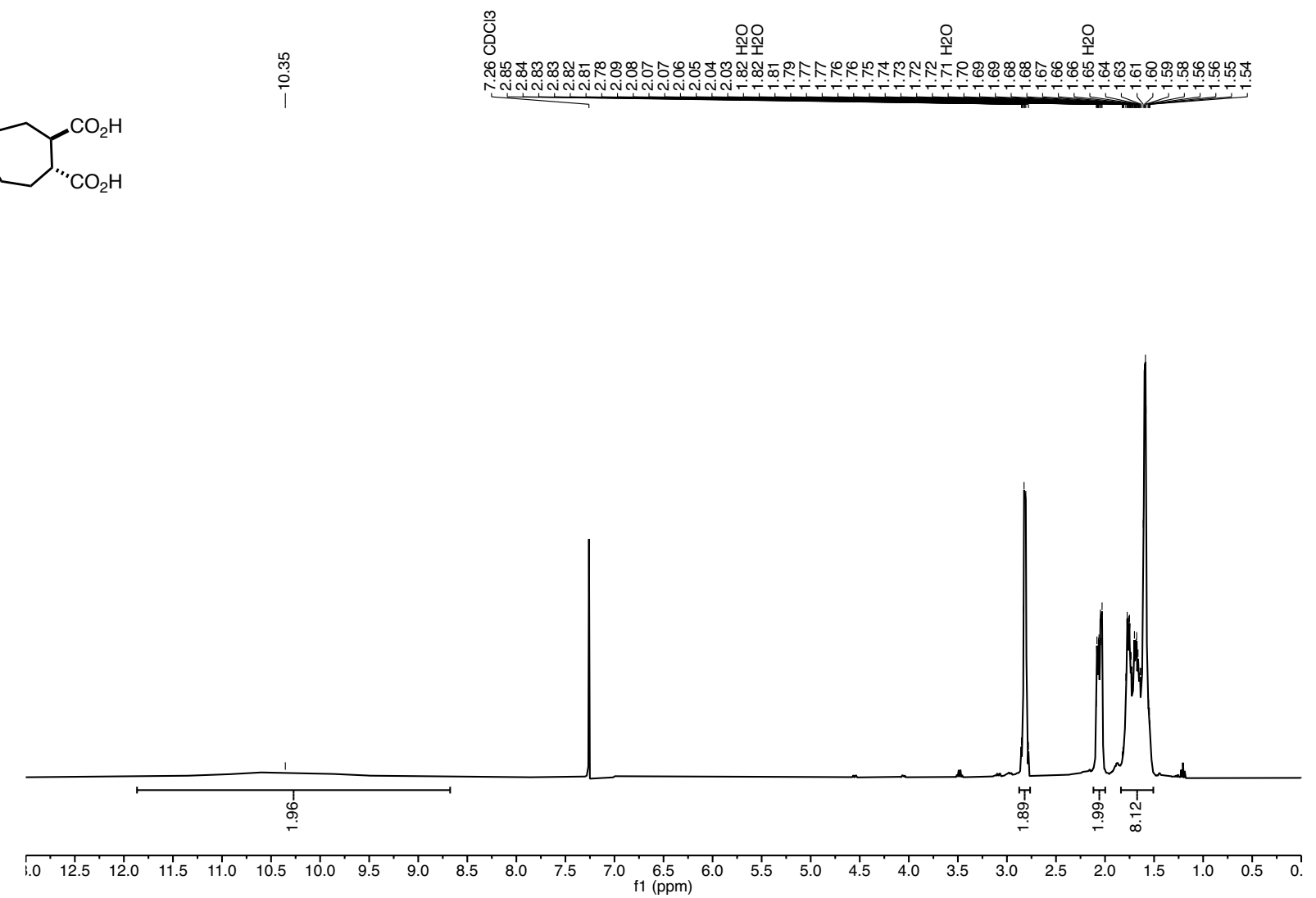

${ }^{13} \mathrm{C}$ NMR (101 MHz, $\left.\mathrm{CDCl}_{3}\right)$

$\sum_{\cdots \mathrm{CO}_{2} \mathrm{H}}^{\mathrm{CO}_{2} \mathrm{H}}$

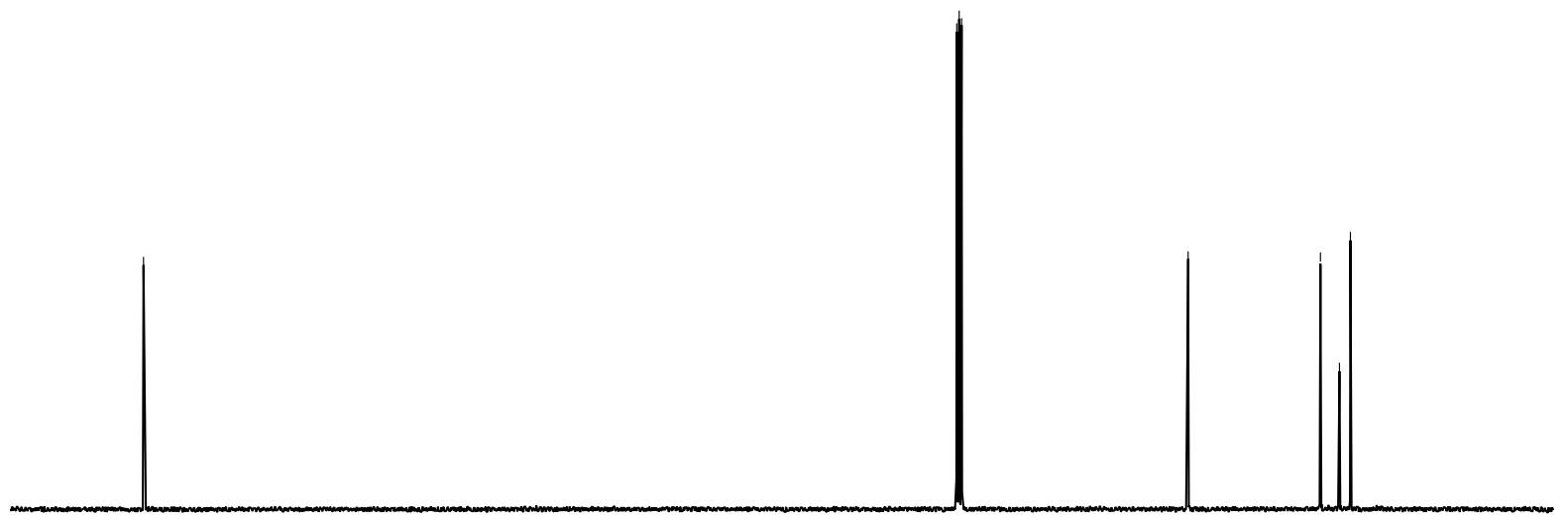

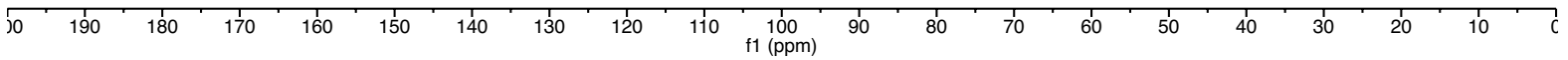


Dibenzyl ester $(+)-\mathbf{S 2}$

${ }^{1} \mathrm{H}$ NMR (400 MHz, $\mathrm{CDCl}_{3}$ )

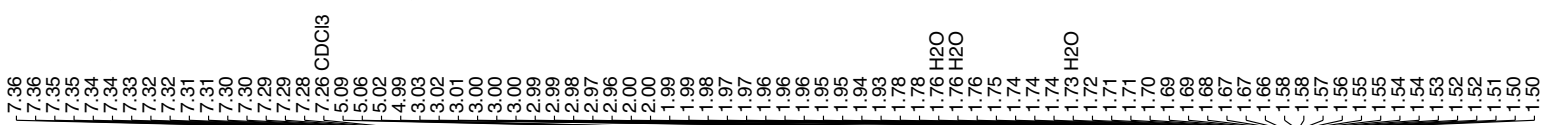

$\int_{\mathrm{CO}_{2} \mathrm{Bn}}^{\cdot \mathrm{CO}_{2} \mathrm{Bn}}$

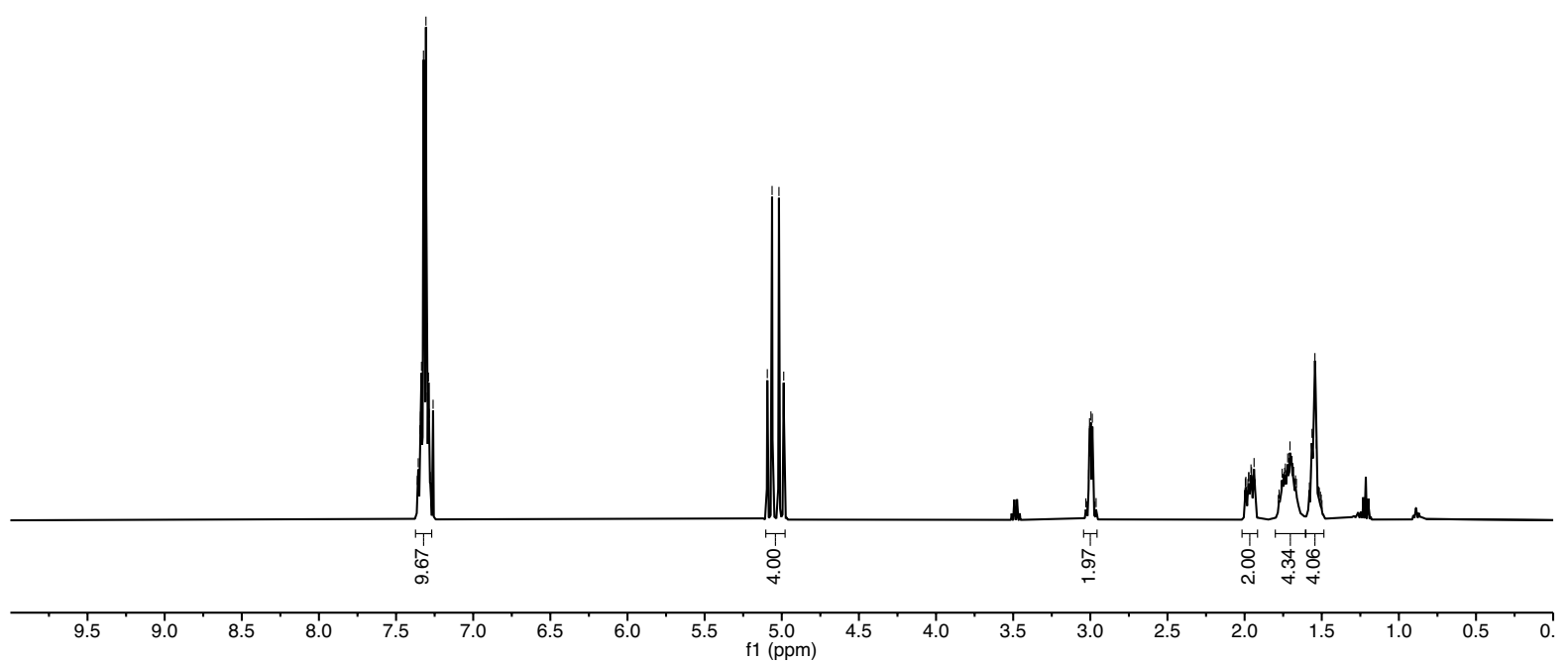

${ }^{13} \mathrm{C}$ NMR (101 MHz, $\left.\mathrm{CDCl}_{3}\right)$

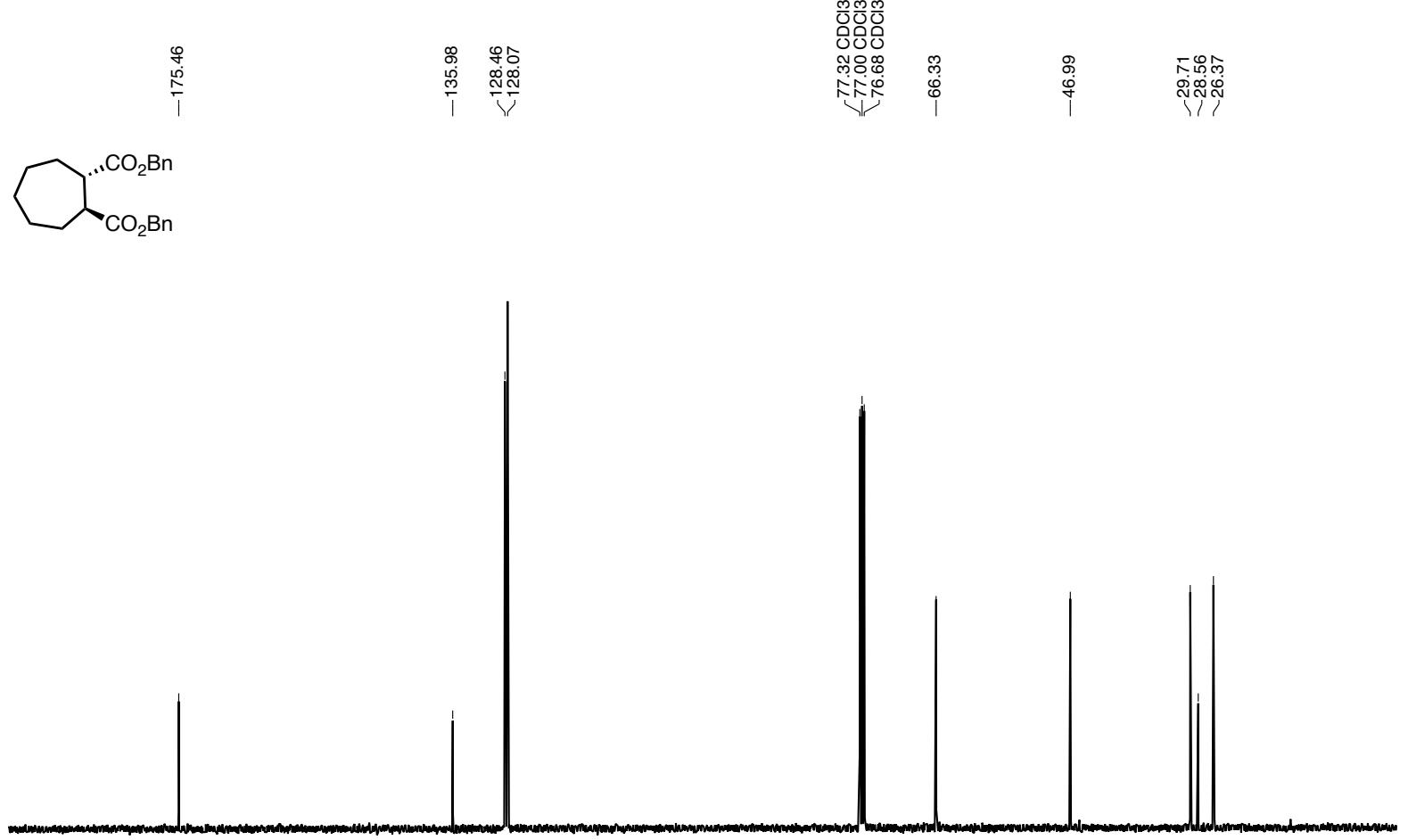

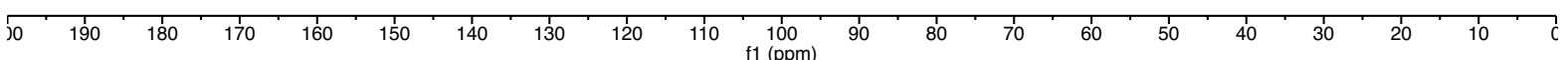


Dimethyl ester (-)-6

\section{${ }^{1} \mathrm{H}$ NMR (400 MHz, $\mathrm{CDCl}_{3}$ )}

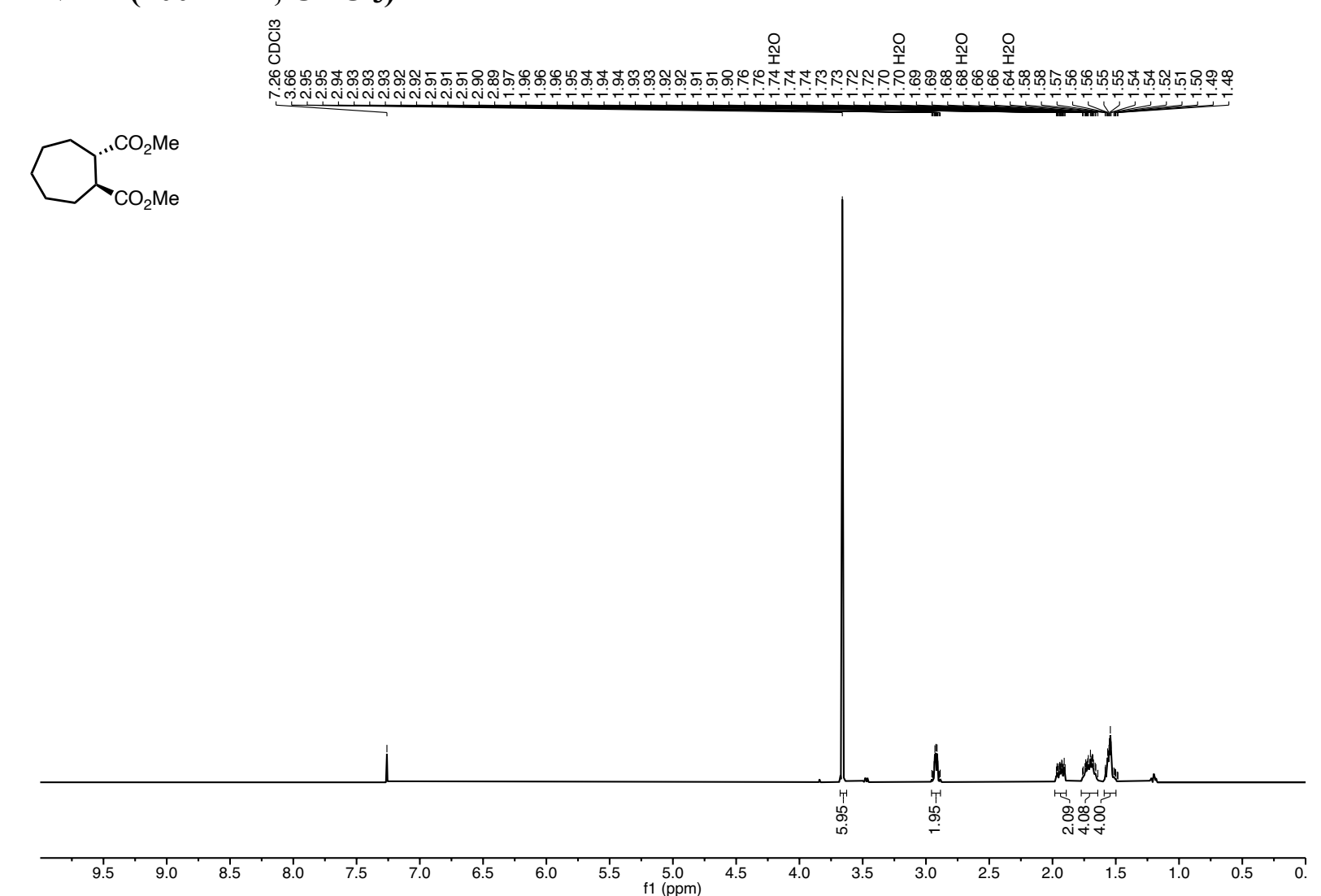

${ }^{13} \mathrm{C}$ NMR (101 MHz, $\left.\mathrm{CDCl}_{3}\right)$
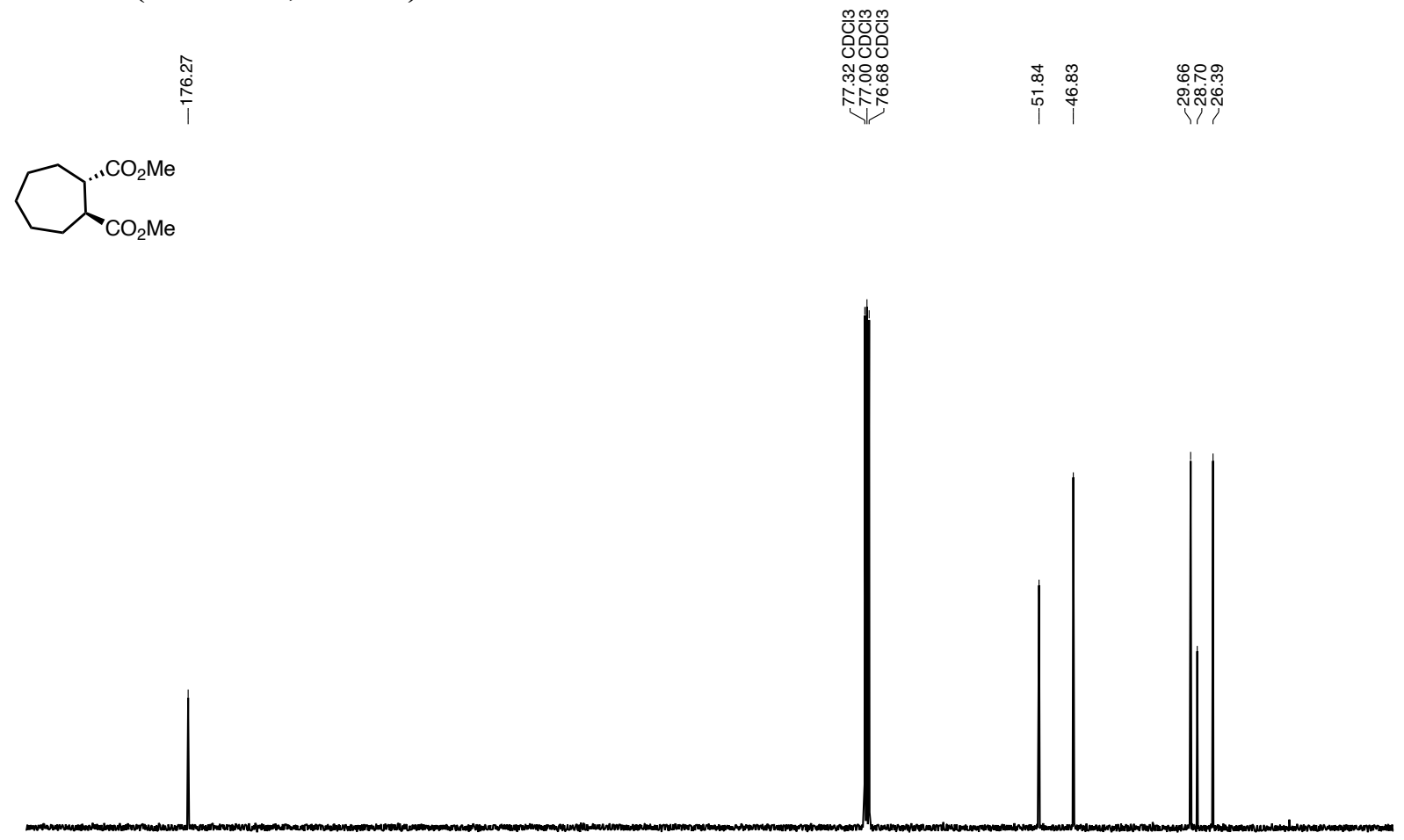

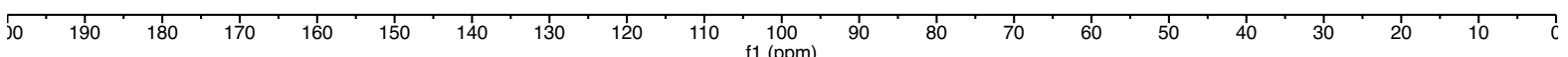


Dimesylate $(+)-7$

\section{${ }^{1} \mathrm{H}$ NMR (400 $\mathrm{MHz}, \mathrm{CDCl}_{3}$ )}
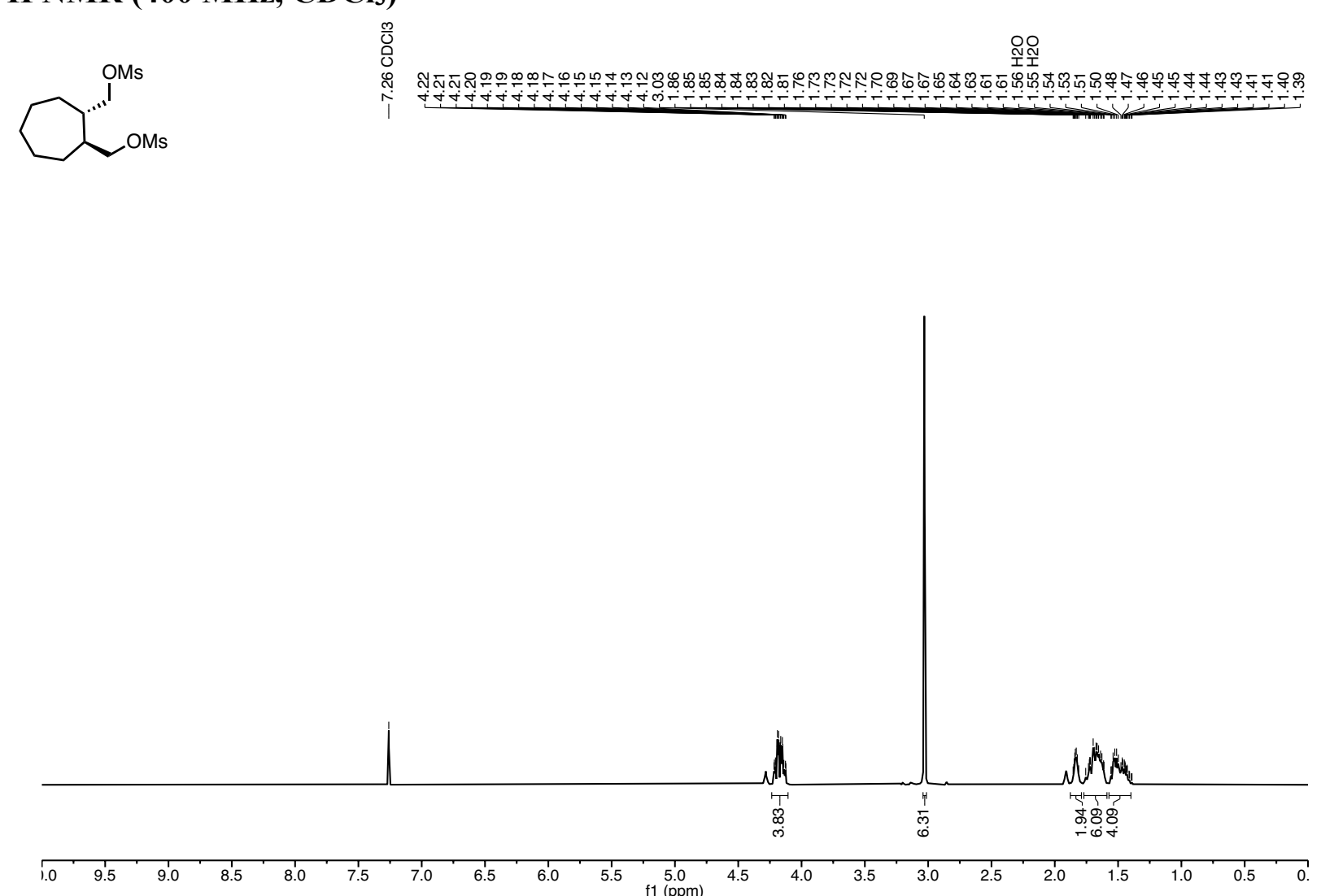

${ }^{13} \mathrm{C}$ NMR (101 MHz, $\left.\mathrm{CDCl}_{3}\right)$
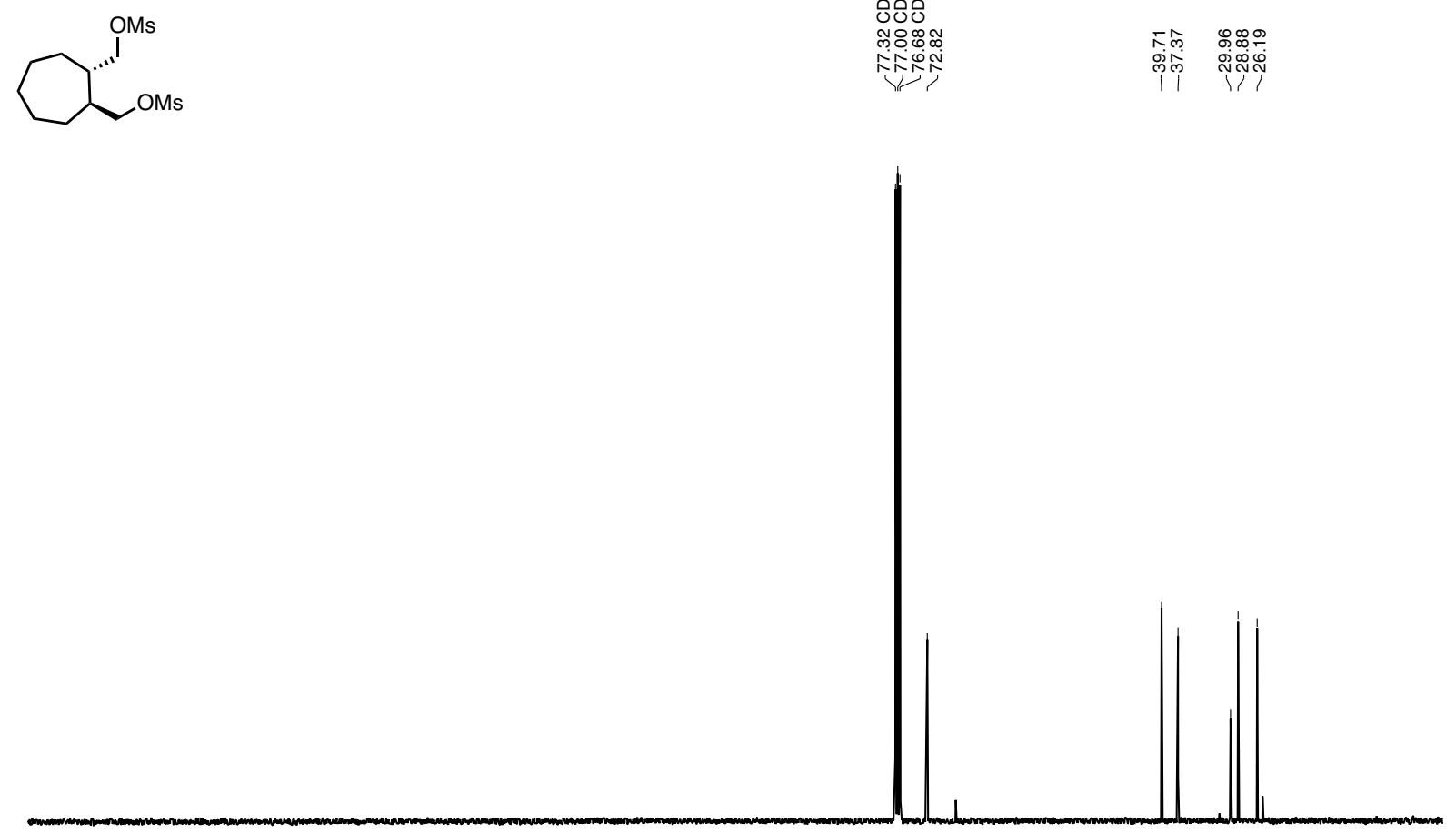

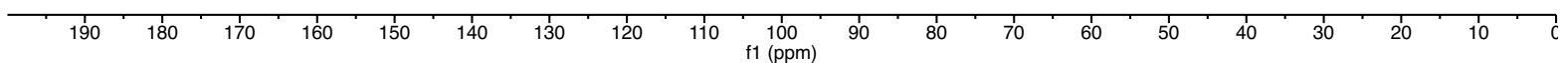


Dinitrile $(+)-8$

${ }^{1} \mathrm{H}$ NMR (400 MHz, $\mathrm{CDCl}_{3}$ )
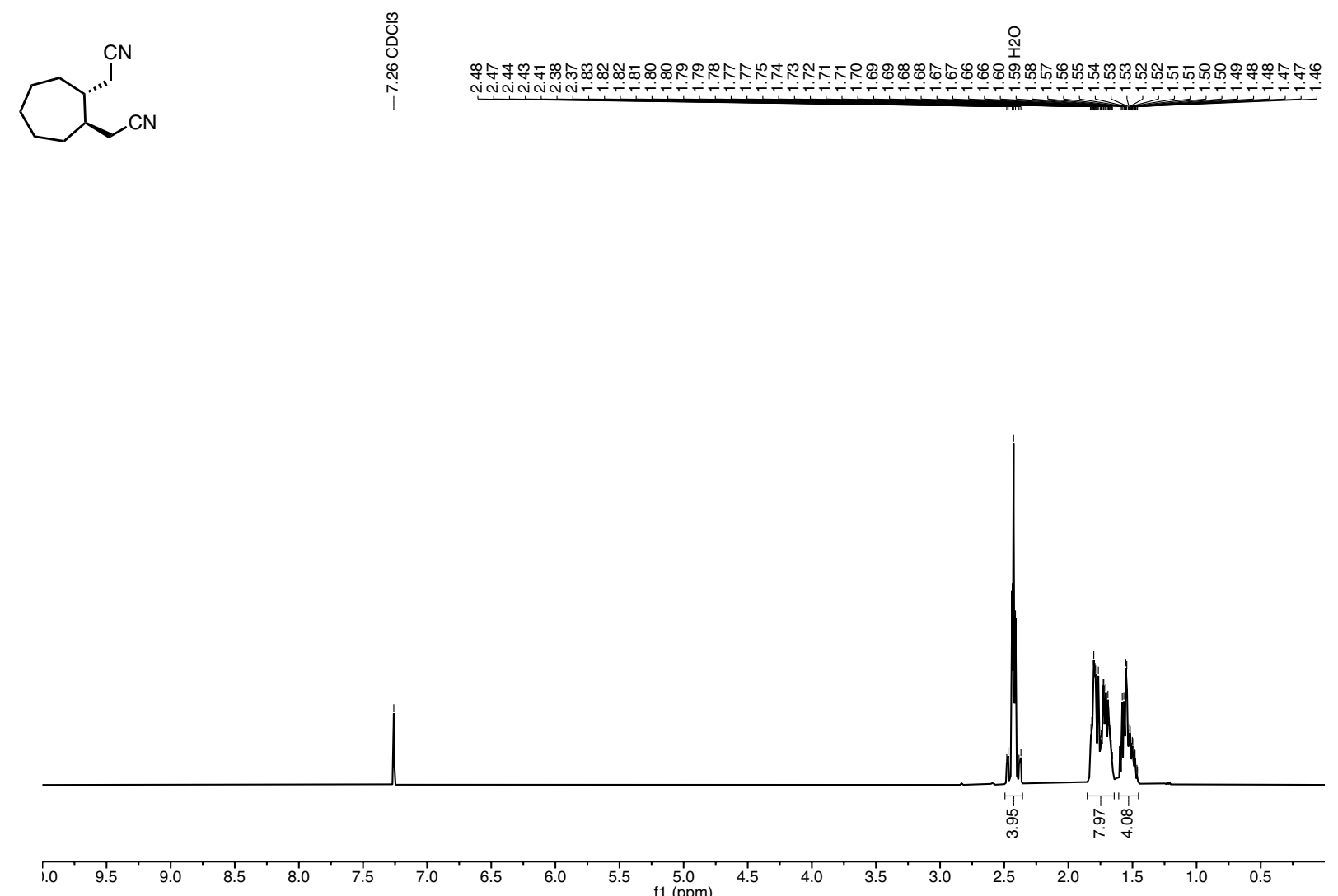

${ }^{13} \mathrm{C}$ NMR (101 MHz, $\left.\mathrm{CDCl}_{3}\right)$
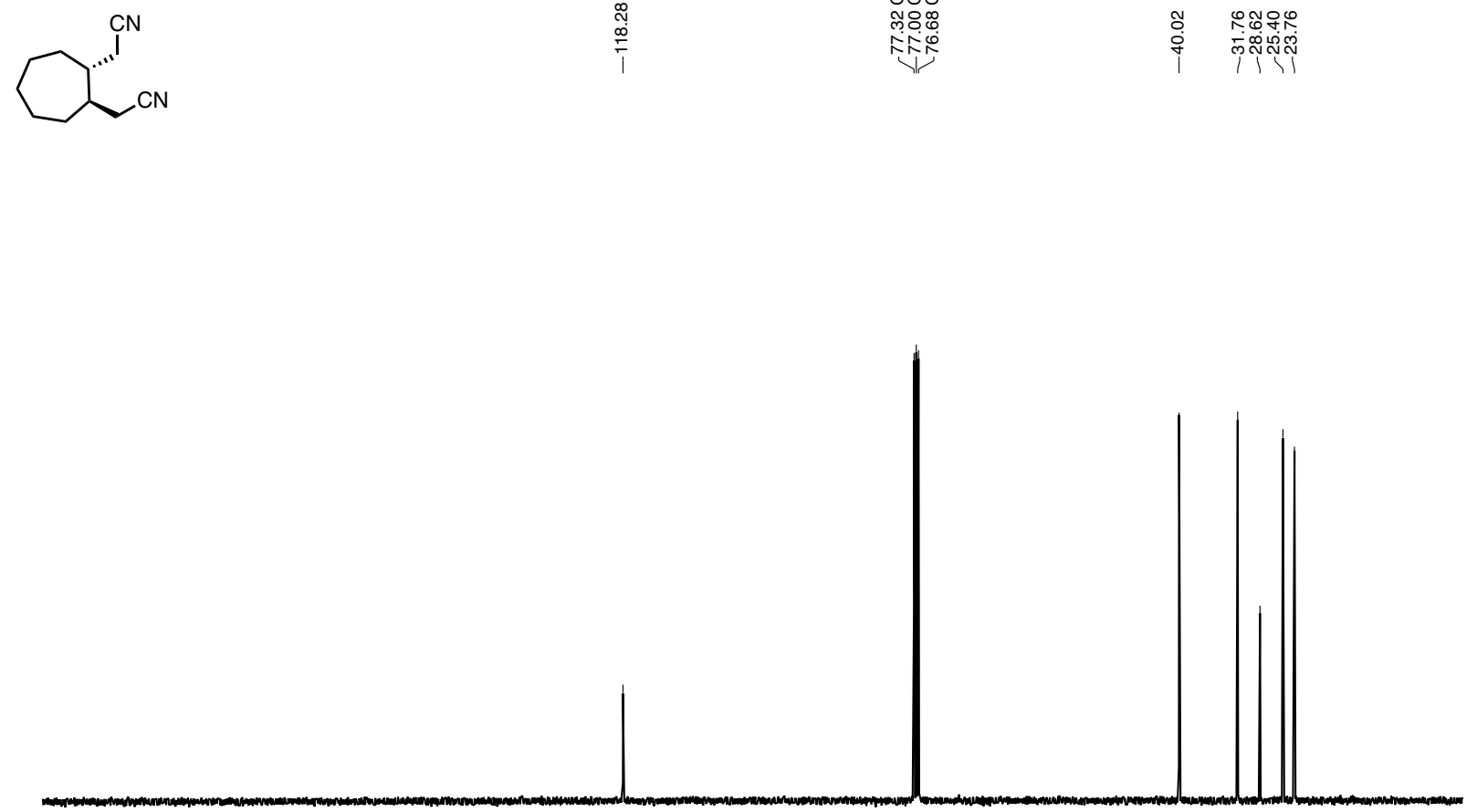

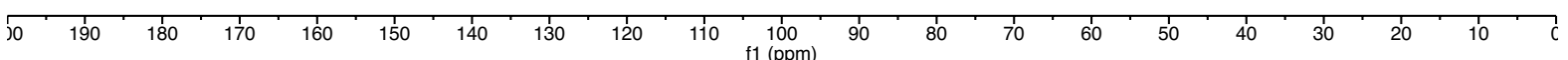


Saturated ketone (-)-9

${ }^{1} \mathrm{H}$ NMR (400 MHz, $\left.\mathrm{CDCl}_{3}\right)$
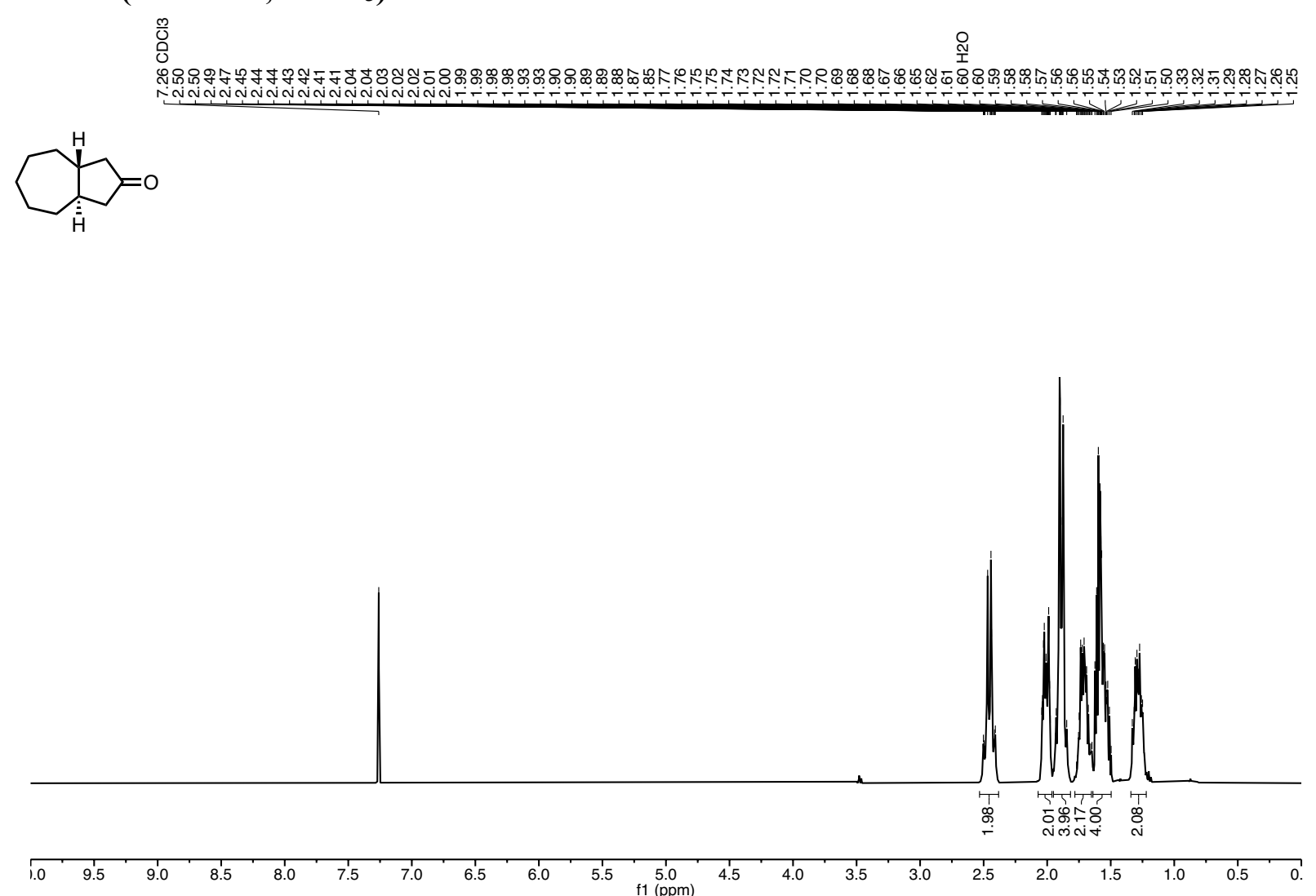

${ }^{13} \mathrm{C}$ NMR (101 MHz, $\left.\mathrm{CDCl}_{3}\right)$
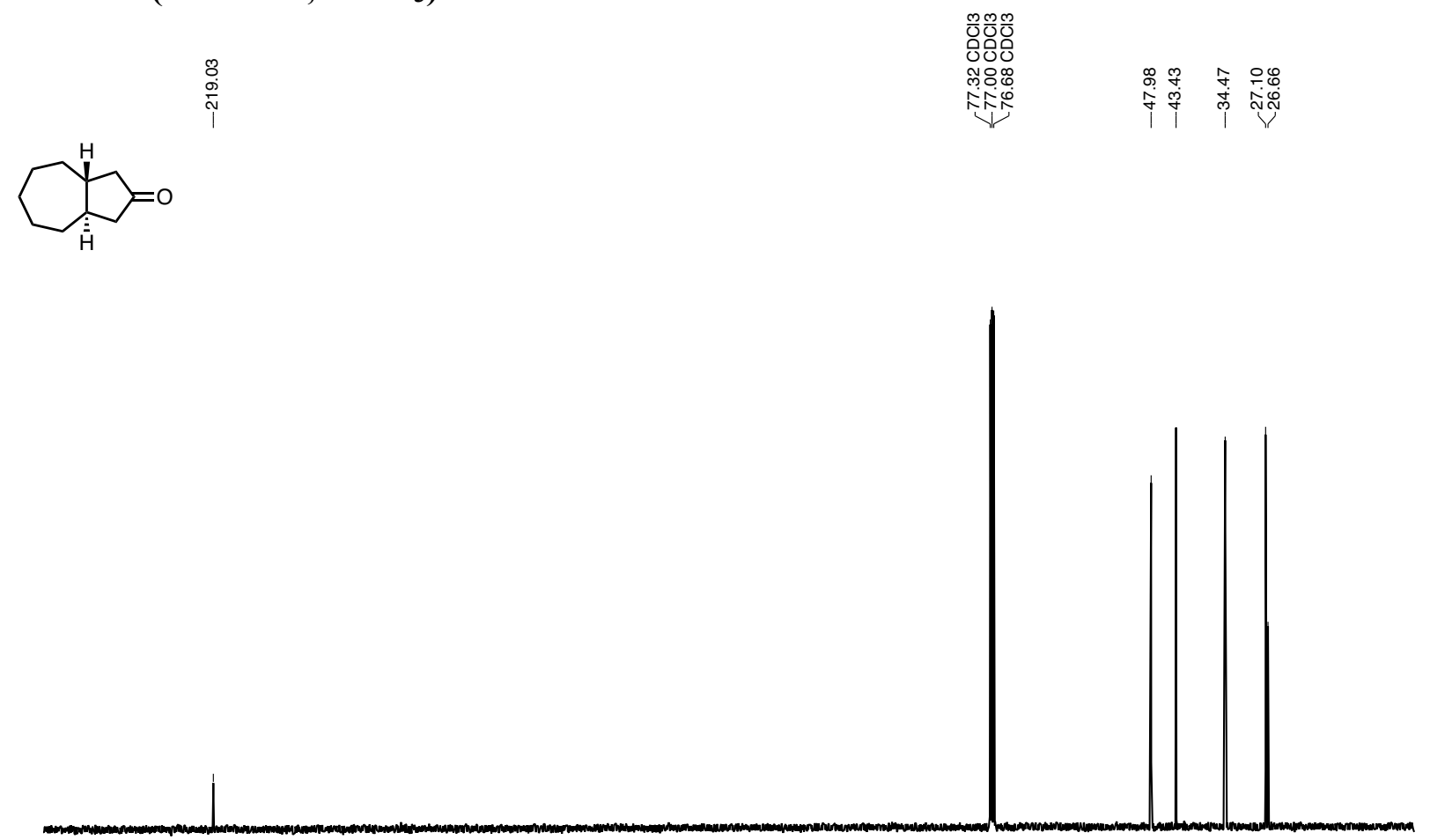

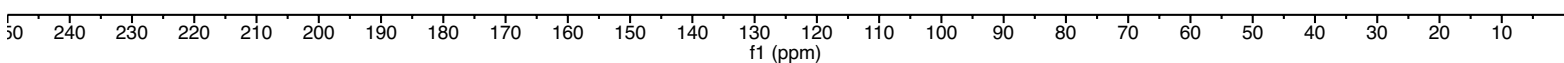


trans-Perhydroazulene (+)-1

${ }^{1} \mathrm{H}$ NMR (400 $\mathrm{MHz}, \mathrm{CDCl}_{3}$ )<smiles>C1CCC2CCCC2CC1</smiles>

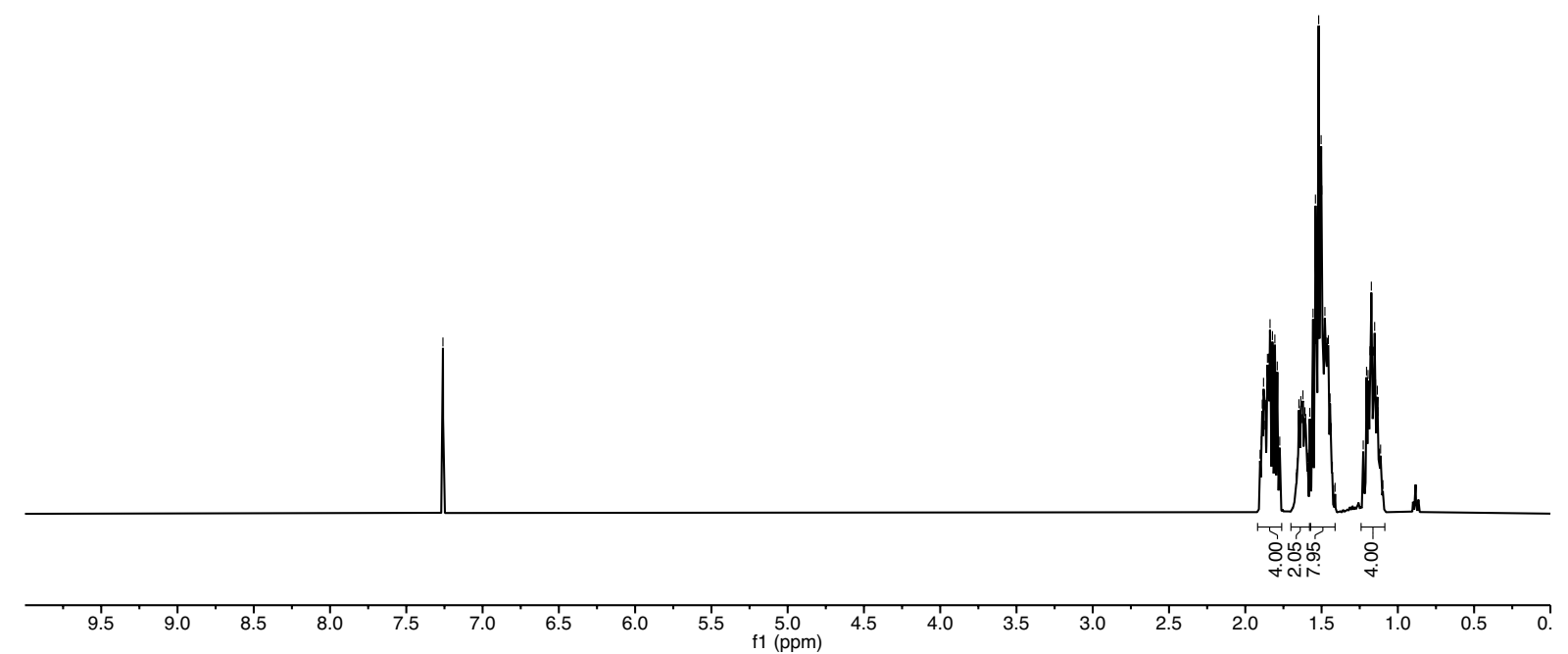

${ }^{13} \mathrm{C}$ NMR (101 MHz, $\left.\mathrm{CDCl}_{3}\right)$<smiles>C1CC[C@@H]2CCC[C@@H]2C1</smiles>

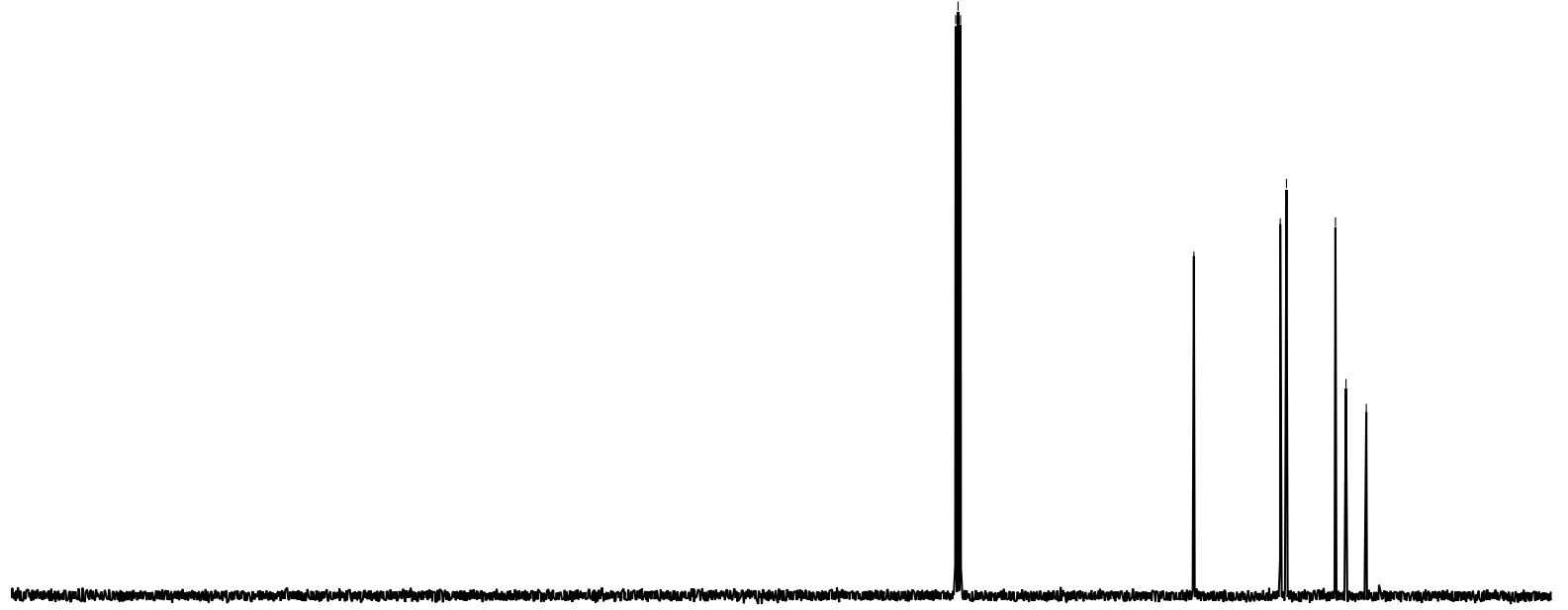

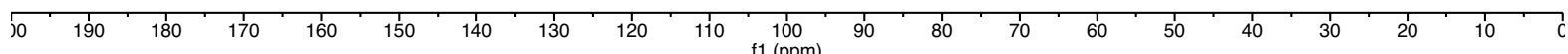




\section{X-ray Crystallographic Analysis}

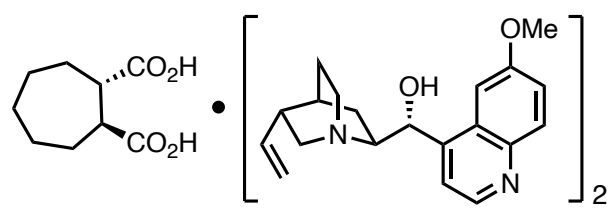

$(-)-5$

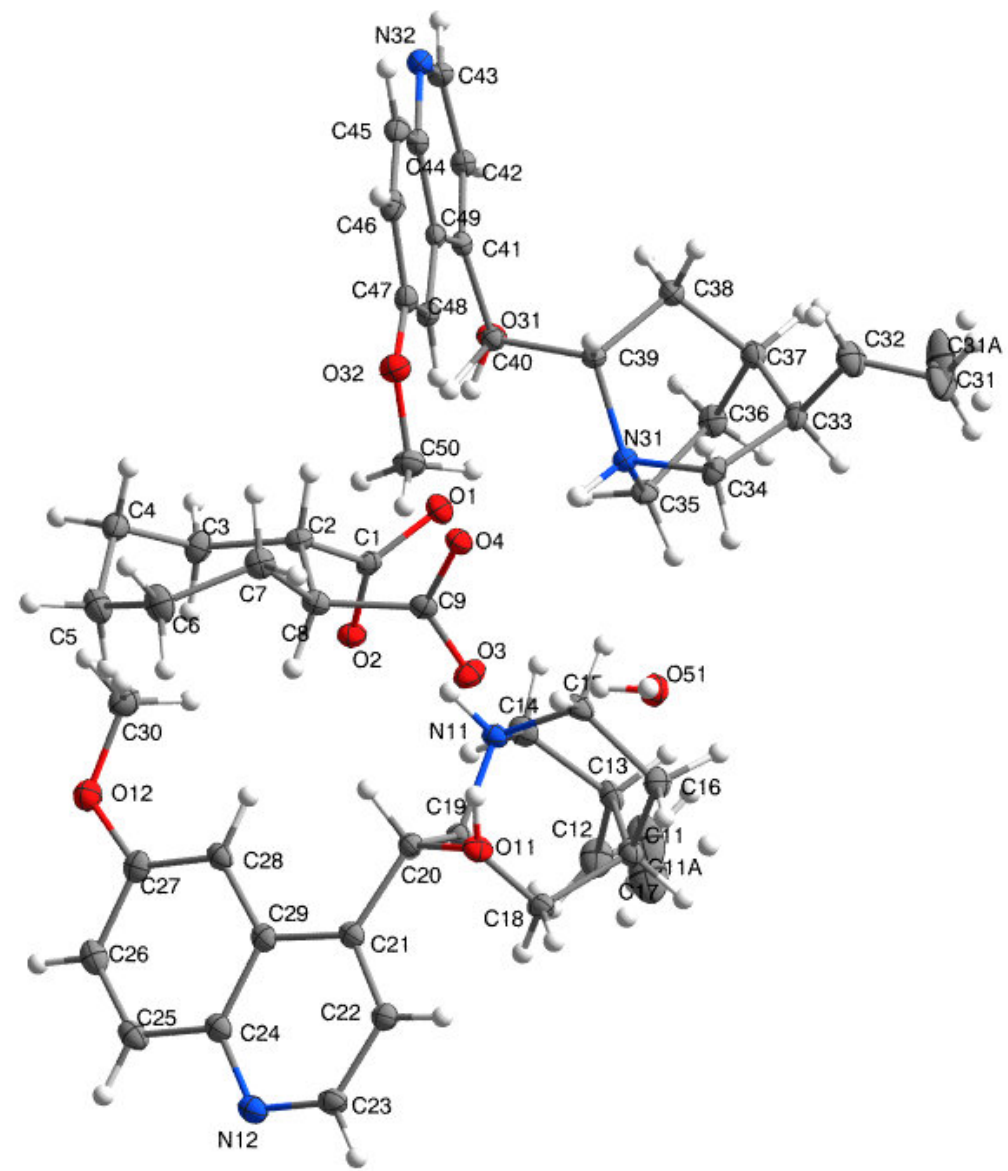

Figure S1. Thermal ellipsoid plot of (-)-5. Thermal ellipsoids are set at the 50\% probability level. Only nonhydrogen atoms are labeled for clarity. 
Crystallization method

Slow evaporation (solvent: ethanol)

\section{Experimental}

Diffraction data for (-)-5 were collected at low temperatures $(100 \mathrm{~K})$ using $\varphi$ - and $\omega$-scans on a BRUKER D8 Venture system equipped with dual I $\mu$ S microfocus sources, a PHOTON100 detector and an OXFORD CRYOSYSTEMS 700 low temperature system. $\mathrm{Cu}-\mathrm{K}_{\alpha}$ radiation with wavelength $1.54178 \AA$ and a collimating Quazar multilayer mirror were used. Semi-empirical absorption correction from equivalents was applied using SADABS-2016/2 ${ }^{4}$ and the structure was solved by direct methods using SHELXT2014/5 orthorhombic space group $P 2{ }_{1} 2{ }_{1} 2$. Refinement was performed against $F^{2}$ on all data by full-matrix least squares using SHELXL2018/3. ${ }^{6}$ All non-hydrogen atoms were refined anisotropically, N-H and O-H hydrogen atoms were located in the Fourier synthesis and set to ideal distances, and $\mathrm{C}-\mathrm{H}$ hydrogen atoms were positioned at geometrically calculated positions and refined using a riding model. The isotropic displacement parameters of all hydrogen atoms were fixed to $1.2 \mathrm{x}$ or $1.5 \mathrm{x}\left(\mathrm{CH}_{3}\right.$ and $\mathrm{OH}$ hydrogens $)$ the $\mathrm{U}_{\text {eq }}$ value of the atoms they are linked to. The asymmetric unit contains (-)-5 crystallized with one water molecule. Both allylic groups were found to be disordered over two positions. The disorder was modeled with the help of same distance restraints on 1,2 and 1,3 distances and similarity restraints on the anisotropic displacement parameters of the disordered atoms and the atoms next to them. ${ }^{7}$ Two disordered atoms very close to each other were set to the same anisotropic displacement parameters. The disorder rations were allowed to refine freely and converged to 0.71(3) and 0.65(6). Anomalous dispersion was used to determine the absolute structure. A Parsons parameter of 0.025(15) confirmed the correct assignment of the absolute structure. $^{8}$

The crystallographic data has been deposited with the Cambridge Crystallographic Data Centre as CCDC No. 1988737 and can be obtained free of charge. ${ }^{9}$ 
Table S1. Crystal data and structure refinement for (-)-5.

CCDC No

Empirical formula

Formula weight

Temperature

Wavelength

Crystal system

Space group

Unit cell dimensions

Volume

Z

Density (calculated)

Absorption coefficient

$\mathrm{F}(000)$

Crystal size

Theta range for data collection

Index ranges

Reflections collected

Independent reflections

Completeness to theta $=67.679^{\circ}$

Absorption correction

Refinement method

Data / restraints / parameters

Goodness-of-fit on $F^{2}$

Final $\mathrm{R}$ indices $[\mathrm{I}>2 \sigma(\mathrm{I})]$

$\mathrm{R}$ indices (all data)

Absolute structure parameter

Extinction coefficient

Largest diff. peak and hole
1988737

$\mathrm{C}_{49} \mathrm{H}_{64} \mathrm{~N}_{4} \mathrm{O}_{9}$

853.04

$100(2) \mathrm{K}$

$1.54178 \AA$

Orthorhombic

$P 2{ }_{12} 2$

$$
\begin{array}{ll}
\mathrm{a}=20.8371(6) \AA & \alpha=90^{\circ} . \\
\mathrm{b}=30.5188(8) \AA & \beta=90^{\circ} . \\
\mathrm{c}=6.7745(2) \AA & \gamma=90^{\circ} .
\end{array}
$$$$
\text { 4308.1(2) } \AA^{3}
$$$$
4
$$

$1.315 \mathrm{Mg} / \mathrm{m}^{3}$

$0.731 \mathrm{~mm}^{-1}$

1832

$0.630 \times 0.502 \times 0.265 \mathrm{~mm}^{3}$

2.568 to $74.456^{\circ}$.

$-26<=\mathrm{h}<=26,-38<=\mathrm{k}<=38,-8<=\mathrm{l}<=8$

370094

$8830[\mathrm{R}(\mathrm{int})=0.0445]$

$100.0 \%$

Semi-empirical from equivalents

Full-matrix least-squares on $F^{2}$

8830 / 30 / 594

1.062

$\mathrm{R}_{1}=0.0263, \mathrm{wR}_{2}=0.0759$

$\mathrm{R}_{1}=0.0266, \mathrm{wR}_{2}=0.0762$

$0.025(15)$

$0.00286(15)$

0.185 and -0.200 e. $\AA^{-3}$ 
Table S2. Atomic coordinates $\left(\times 10^{4}\right)$ and equivalent isotropic displacement parameters $\left(\AA^{2} \times 10^{3}\right)$ for $(-)-5$. $U\left(\right.$ eq) is defined as one third of the trace of the orthogonalized $U^{\mathrm{ij}}$ tensor.

\begin{tabular}{|c|c|c|c|c|}
\hline & $\mathrm{x}$ & $\mathrm{y}$ & $\mathrm{z}$ & $\mathrm{U}(\mathrm{eq})$ \\
\hline $\mathrm{O}(1)$ & $2670(1)$ & $7296(1)$ & $4907(2)$ & $17(1)$ \\
\hline $\mathrm{O}(2)$ & $2252(1)$ & $7965(1)$ & $5250(2)$ & $16(1)$ \\
\hline $\mathrm{O}(3)$ & $2470(1)$ & $7721(1)$ & $477(2)$ & $19(1)$ \\
\hline $\mathrm{O}(4)$ & $2153(1)$ & $7028(1)$ & $51(2)$ & $15(1)$ \\
\hline $\mathrm{C}(1)$ & $2212(1)$ & $7566(1)$ & $4793(2)$ & $13(1)$ \\
\hline$C(2)$ & $1564(1)$ & $7397(1)$ & $4047(2)$ & $14(1)$ \\
\hline$C(3)$ & $1041(1)$ & $7570(1)$ & $5428(2)$ & $19(1)$ \\
\hline$C(4)$ & $345(1)$ & $7445(1)$ & $4982(2)$ & $19(1)$ \\
\hline$C(5)$ & $64(1)$ & $7687(1)$ & $3212(2)$ & $22(1)$ \\
\hline$C(6)$ & $223(1)$ & $7489(1)$ & $1196(2)$ & $24(1)$ \\
\hline$C(7)$ & $873(1)$ & $7257(1)$ & $1029(2)$ & $17(1)$ \\
\hline$C(8)$ & $1452(1)$ & $7512(1)$ & $1833(2)$ & $14(1)$ \\
\hline $\mathrm{C}(9)$ & $2072(1)$ & $7414(1)$ & $672(2)$ & $13(1)$ \\
\hline $\mathrm{O}(11)$ & $2755(1)$ & $8581(1)$ & $399(2)$ & $16(1)$ \\
\hline $\mathrm{O}(12)$ & 988(1) & $9074(1)$ & $8310(2)$ & $19(1)$ \\
\hline $\mathrm{N}(11)$ & $3339(1)$ & $8385(1)$ & $4599(2)$ & $13(1)$ \\
\hline $\mathrm{N}(12)$ & $1655(1)$ & $10023(1)$ & $1666(2)$ & $17(1)$ \\
\hline $\mathrm{C}(11)$ & $5038(3)$ & 9170(4) & $7785(15)$ & $41(2)$ \\
\hline$C(11 A)$ & $5038(8)$ & $9289(6)$ & $7350(30)$ & $41(2)$ \\
\hline $\mathrm{C}(12)$ & $4526(1)$ & $9146(1)$ & $6572(2)$ & $25(1)$ \\
\hline$C(13)$ & $4384(1)$ & $8736(1)$ & $5431(2)$ & $16(1)$ \\
\hline$C(14)$ & $3778(1)$ & $8504(1)$ & $6268(2)$ & $17(1)$ \\
\hline$C(15)$ & $3703(1)$ & $8103(1)$ & $3157(2)$ & $16(1)$ \\
\hline$C(16)$ & $4229(1)$ & $8378(1)$ & $2151(2)$ & $18(1)$ \\
\hline$C(17)$ & $4280(1)$ & $8820(1)$ & $3210(2)$ & $16(1)$ \\
\hline $\mathrm{C}(18)$ & $3651(1)$ & $9071(1)$ & $2866(2)$ & $16(1)$ \\
\hline$C(19)$ & $3083(1)$ & $8808(1)$ & $3725(2)$ & $13(1)$ \\
\hline$C(20)$ & $2527(1)$ & $8733(1)$ & $2247(2)$ & $13(1)$ \\
\hline$C(21)$ & $2188(1)$ & $9171(1)$ & $2020(2)$ & $14(1)$ \\
\hline$C(22)$ & $2308(1)$ & $9432(1)$ & $410(2)$ & $15(1)$ \\
\hline$C(23)$ & $2027(1)$ & $9852(1)$ & $296(2)$ & $17(1)$ \\
\hline$C(24)$ & $1525(1)$ & $9765(1)$ & $3273(2)$ & $16(1)$ \\
\hline$C(25)$ & $1135(1)$ & $9948(1)$ & $4789(2)$ & $18(1)$ \\
\hline$C(26)$ & 982(1) & $9712(1)$ & $6431(2)$ & $18(1)$ \\
\hline$C(27)$ & $1201(1)$ & $9273(1)$ & $6639(2)$ & $16(1)$ \\
\hline
\end{tabular}




\begin{tabular}{|c|c|c|c|c|}
\hline$C(28)$ & $1589(1)$ & $9087(1)$ & $5232(2)$ & $15(1)$ \\
\hline$C(29)$ & $1768(1)$ & $9332(1)$ & $3522(2)$ & $14(1)$ \\
\hline$C(30)$ & $1172(1)$ & $8630(1)$ & $8619(2)$ & $22(1)$ \\
\hline $\mathrm{O}(31)$ & $2790(1)$ & $6415(1)$ & $4738(1)$ & $15(1)$ \\
\hline $\mathrm{O}(32)$ & 998(1) & $5906(1)$ & $-3084(2)$ & $18(1)$ \\
\hline $\mathrm{N}(31)$ & $3310(1)$ & $6632(1)$ & $468(2)$ & $13(1)$ \\
\hline $\mathrm{N}(32)$ & $1719(1)$ & $4962(1)$ & $3509(2)$ & $16(1)$ \\
\hline $\mathrm{C}(31)$ & $5011(5)$ & $5879(5)$ & $-2934(19)$ & $32(2)$ \\
\hline$C(31 \mathrm{~A})$ & $5012(7)$ & $5803(12)$ & $-2730(40)$ & $41(5)$ \\
\hline$C(32)$ & $4496(1)$ & $5898(1)$ & $-1769(2)$ & $22(1)$ \\
\hline$C(33)$ & $4348(1)$ & $6297(1)$ & $-549(2)$ & $15(1)$ \\
\hline$C(34)$ & $3722(1)$ & $6520(1)$ & $-1281(2)$ & $16(1)$ \\
\hline$C(35)$ & $3701(1)$ & $6910(1)$ & $1864(2)$ & $15(1)$ \\
\hline$C(36)$ & $4233(1)$ & $6628(1)$ & $2798(2)$ & $17(1)$ \\
\hline$C(37)$ & $4275(1)$ & $6194(1)$ & $1674(2)$ & $15(1)$ \\
\hline $\mathrm{C}(38)$ & $3656(1)$ & $5937(1)$ & $2067(2)$ & $15(1)$ \\
\hline C(39) & $3072(1)$ & $6204(1)$ & $1343(2)$ & $12(1)$ \\
\hline$C(40)$ & $2539(1)$ & $6267(1)$ & $2912(2)$ & $12(1)$ \\
\hline$C(41)$ & $2220(1)$ & $5823(1)$ & $3161(2)$ & $13(1)$ \\
\hline$C(42)$ & $2351(1)$ & $5564(1)$ & $4766(2)$ & $15(1)$ \\
\hline$C(43)$ & $2084(1)$ & $5139(1)$ & $4881(2)$ & $16(1)$ \\
\hline$C(44)$ & $1578(1)$ & $5217(1)$ & $1905(2)$ & $14(1)$ \\
\hline$C(45)$ & 1194(1) & $5028(1)$ & $390(2)$ & $16(1)$ \\
\hline$C(46)$ & $1025(1)$ & $5263(1)$ & $-1240(2)$ & $17(1)$ \\
\hline$C(47)$ & $1224(1)$ & $5706(1)$ & $-1432(2)$ & $15(1)$ \\
\hline$C(48)$ & $1607(1)$ & $5899(1)$ & $-26(2)$ & $14(1)$ \\
\hline$C(49)$ & 1801(1) & $5655(1)$ & $1666(2)$ & $13(1)$ \\
\hline$C(50)$ & $1185(1)$ & $6349(1)$ & $-3404(2)$ & $20(1)$ \\
\hline $\mathrm{O}(51)$ & $3470(1)$ & $7548(1)$ & $-2060(2)$ & $20(1)$ \\
\hline
\end{tabular}


Table S3. Bond lengths $[\AA]$ and angles $\left[{ }^{\circ}\right]$ for $(-)-5$.

\begin{tabular}{|c|c|c|c|}
\hline $\mathrm{O}(1)-\mathrm{C}(1)$ & $1.2642(16)$ & $C(11 A)-C(12)$ & $1.268(13)$ \\
\hline $\mathrm{O}(2)-\mathrm{C}(1)$ & $1.2590(16)$ & $\mathrm{C}(11 \mathrm{~A})-\mathrm{H}(11 \mathrm{C})$ & 0.9500 \\
\hline $\mathrm{O}(3)-\mathrm{C}(9)$ & $1.2592(16)$ & $\mathrm{C}(11 \mathrm{~A})-\mathrm{H}(11 \mathrm{D})$ & 0.9500 \\
\hline $\mathrm{O}(4)-\mathrm{C}(9)$ & $1.2600(16)$ & $C(12)-C(13)$ & $1.4999(19)$ \\
\hline$C(1)-C(2)$ & $1.5317(18)$ & $\mathrm{C}(12)-\mathrm{H}(12)$ & 0.9500 \\
\hline$C(2)-C(3)$ & $1.5303(18)$ & $\mathrm{C}(12)-\mathrm{H}(12 \mathrm{~A})$ & 0.9500 \\
\hline$C(2)-C(8)$ & $1.5582(17)$ & $\mathrm{C}(13)-\mathrm{C}(17)$ & $1.541(2)$ \\
\hline $\mathrm{C}(2)-\mathrm{H}(2)$ & 1.0000 & $C(13)-C(14)$ & $1.5545(19)$ \\
\hline$C(3)-C(4)$ & $1.5285(18)$ & $\mathrm{C}(13)-\mathrm{H}(13)$ & 1.0000 \\
\hline $\mathrm{C}(3)-\mathrm{H}(3 \mathrm{~A})$ & 0.9900 & $\mathrm{C}(14)-\mathrm{H}(14 \mathrm{~A})$ & 0.9900 \\
\hline $\mathrm{C}(3)-\mathrm{H}(3 \mathrm{AB})$ & 0.9900 & $\mathrm{C}(14)-\mathrm{H}(14 \mathrm{~B})$ & 0.9900 \\
\hline$C(4)-C(5)$ & $1.525(2)$ & $C(15)-C(16)$ & $1.5388(19)$ \\
\hline $\mathrm{C}(4)-\mathrm{H}(4 \mathrm{~A})$ & 0.9900 & $\mathrm{C}(15)-\mathrm{H}(15 \mathrm{~A})$ & 0.9900 \\
\hline $\mathrm{C}(4)-\mathrm{H}(4 \mathrm{AB})$ & 0.9900 & $\mathrm{C}(15)-\mathrm{H}(15 \mathrm{~B})$ & 0.9900 \\
\hline$C(5)-C(6)$ & $1.529(2)$ & $C(16)-C(17)$ & $1.5332(18)$ \\
\hline $\mathrm{C}(5)-\mathrm{H}(5 \mathrm{~A})$ & 0.9900 & $\mathrm{C}(16)-\mathrm{H}(16 \mathrm{~A})$ & 0.9900 \\
\hline $\mathrm{C}(5)-\mathrm{H}(5 \mathrm{AB})$ & 0.9900 & $\mathrm{C}(16)-\mathrm{H}(16 \mathrm{~B})$ & 0.9900 \\
\hline$C(6)-C(7)$ & $1.5319(19)$ & $\mathrm{C}(17)-\mathrm{C}(18)$ & $1.5358(18)$ \\
\hline $\mathrm{C}(6)-\mathrm{H}(6 \mathrm{~A})$ & 0.9900 & $\mathrm{C}(17)-\mathrm{H}(17)$ & 1.0000 \\
\hline $\mathrm{C}(6)-\mathrm{H}(6 \mathrm{AB})$ & 0.9900 & $\mathrm{C}(18)-\mathrm{C}(19)$ & $1.5445(18)$ \\
\hline $\mathrm{C}(7)-\mathrm{C}(8)$ & $1.5358(17)$ & $\mathrm{C}(18)-\mathrm{H}(18 \mathrm{~A})$ & 0.9900 \\
\hline $\mathrm{C}(7)-\mathrm{H}(7 \mathrm{~A})$ & 0.9900 & $\mathrm{C}(18)-\mathrm{H}(18 \mathrm{~B})$ & 0.9900 \\
\hline $\mathrm{C}(7)-\mathrm{H}(7 \mathrm{AB})$ & 0.9900 & C(19)-C(20) & $1.5486(18)$ \\
\hline $\mathrm{C}(8)-\mathrm{C}(9)$ & $1.5422(18)$ & $\mathrm{C}(19)-\mathrm{H}(19)$ & 1.0000 \\
\hline $\mathrm{C}(8)-\mathrm{H}(8)$ & 1.0000 & $\mathrm{C}(20)-\mathrm{C}(21)$ & $1.5192(17)$ \\
\hline $\mathrm{O}(11)-\mathrm{C}(20)$ & $1.4172(16)$ & $\mathrm{C}(20)-\mathrm{H}(20)$ & 1.0000 \\
\hline $\mathrm{O}(11)-\mathrm{H}(11 \mathrm{O})$ & $0.839(16)$ & $C(21)-C(22)$ & $1.3731(19)$ \\
\hline $\mathrm{O}(12)-\mathrm{C}(27)$ & $1.3595(18)$ & $\mathrm{C}(21)-\mathrm{C}(29)$ & $1.4296(19)$ \\
\hline $\mathrm{O}(12)-\mathrm{C}(30)$ & $1.4234(18)$ & $\mathrm{C}(22)-\mathrm{C}(23)$ & $1.4119(19)$ \\
\hline $\mathrm{N}(11)-\mathrm{C}(14)$ & $1.4994(17)$ & $\mathrm{C}(22)-\mathrm{H}(22)$ & 0.9500 \\
\hline $\mathrm{N}(11)-\mathrm{C}(15)$ & $1.5070(18)$ & $\mathrm{C}(23)-\mathrm{H}(23)$ & 0.9500 \\
\hline $\mathrm{N}(11)-\mathrm{C}(19)$ & $1.5155(16)$ & $\mathrm{C}(24)-\mathrm{C}(25)$ & $1.424(2)$ \\
\hline $\mathrm{N}(11)-\mathrm{H}(11)$ & $0.928(15)$ & $\mathrm{C}(24)-\mathrm{C}(29)$ & $1.4249(18)$ \\
\hline $\mathrm{N}(12)-\mathrm{C}(23)$ & $1.317(2)$ & $C(25)-C(26)$ & $1.363(2)$ \\
\hline $\mathrm{N}(12)-\mathrm{C}(24)$ & $1.3699(19)$ & $\mathrm{C}(25)-\mathrm{H}(25)$ & 0.9500 \\
\hline$C(11)-C(12)$ & $1.350(6)$ & $C(26)-C(27)$ & $1.423(2)$ \\
\hline $\mathrm{C}(11)-\mathrm{H}(11 \mathrm{~A})$ & 0.9500 & $\mathrm{C}(26)-\mathrm{H}(26)$ & 0.9500 \\
\hline $\mathrm{C}(11)-\mathrm{H}(11 \mathrm{~B})$ & 0.9500 & $\mathrm{C}(27)-\mathrm{C}(28)$ & $1.373(2)$ \\
\hline
\end{tabular}




\begin{tabular}{|c|c|c|c|}
\hline $\mathrm{C}(28)-\mathrm{C}(29)$ & $1.429(2)$ & $C(39)-C(40)$ & $1.5486(18)$ \\
\hline $\mathrm{C}(28)-\mathrm{H}(28)$ & 0.9500 & $\mathrm{C}(39)-\mathrm{H}(39)$ & 1.0000 \\
\hline $\mathrm{C}(30)-\mathrm{H}(30 \mathrm{~A})$ & 0.9800 & $C(40)-C(41)$ & $1.5197(17)$ \\
\hline $\mathrm{C}(30)-\mathrm{H}(30 \mathrm{~B})$ & 0.9800 & $\mathrm{C}(40)-\mathrm{H}(40)$ & 1.0000 \\
\hline $\mathrm{C}(30)-\mathrm{H}(30 \mathrm{C})$ & 0.9800 & $\mathrm{C}(41)-\mathrm{C}(42)$ & $1.3714(19)$ \\
\hline $\mathrm{O}(31)-\mathrm{C}(40)$ & $1.4163(16)$ & $C(41)-C(49)$ & $1.4315(19)$ \\
\hline $\mathrm{O}(31)-\mathrm{H}(31 \mathrm{O})$ & $0.852(16)$ & $\mathrm{C}(42)-\mathrm{C}(43)$ & $1.4142(18)$ \\
\hline $\mathrm{O}(32)-\mathrm{C}(47)$ & $1.3592(18)$ & $\mathrm{C}(42)-\mathrm{H}(42)$ & 0.9500 \\
\hline $\mathrm{O}(32)-\mathrm{C}(50)$ & $1.4226(17)$ & $\mathrm{C}(43)-\mathrm{H}(43)$ & 0.9500 \\
\hline $\mathrm{N}(31)-\mathrm{C}(34)$ & $1.5024(17)$ & $C(44)-C(49)$ & $1.4233(18)$ \\
\hline $\mathrm{N}(31)-\mathrm{C}(35)$ & $1.5097(17)$ & $\mathrm{C}(44)-\mathrm{C}(45)$ & $1.424(2)$ \\
\hline $\mathrm{N}(31)-\mathrm{C}(39)$ & $1.5174(16)$ & $C(45)-C(46)$ & $1.362(2)$ \\
\hline $\mathrm{N}(31)-\mathrm{H}(31)$ & $0.889(15)$ & $\mathrm{C}(45)-\mathrm{H}(45)$ & 0.9500 \\
\hline $\mathrm{N}(32)-\mathrm{C}(43)$ & $1.3172(19)$ & $\mathrm{C}(46)-\mathrm{C}(47)$ & $1.4202(19)$ \\
\hline $\mathrm{N}(32)-\mathrm{C}(44)$ & $1.3691(18)$ & $\mathrm{C}(46)-\mathrm{H}(46)$ & 0.9500 \\
\hline $\mathrm{C}(31)-\mathrm{C}(32)$ & $1.334(8)$ & $\mathrm{C}(47)-\mathrm{C}(48)$ & $1.376(2)$ \\
\hline $\mathrm{C}(31)-\mathrm{H}(31 \mathrm{~A})$ & 0.9500 & $\mathrm{C}(48)-\mathrm{C}(49)$ & $1.4269(19)$ \\
\hline $\mathrm{C}(31)-\mathrm{H}(31 \mathrm{~B})$ & 0.9500 & $\mathrm{C}(48)-\mathrm{H}(48)$ & 0.9500 \\
\hline$C(31 A)-C(32)$ & $1.292(14)$ & $\mathrm{C}(50)-\mathrm{H}(50 \mathrm{~A})$ & 0.9800 \\
\hline $\mathrm{C}(31 \mathrm{~A})-\mathrm{H}(31 \mathrm{C})$ & 0.9500 & $\mathrm{C}(50)-\mathrm{H}(50 \mathrm{~B})$ & 0.9800 \\
\hline $\mathrm{C}(31 \mathrm{~A})-\mathrm{H}(31 \mathrm{D})$ & 0.9500 & $\mathrm{C}(50)-\mathrm{H}(50 \mathrm{C})$ & 0.9800 \\
\hline$C(32)-C(33)$ & $1.5016(19)$ & $\mathrm{O}(51)-\mathrm{H}(51 \mathrm{~A})$ & $0.847(18)$ \\
\hline C(32)-H(32) & 0.9500 & $\mathrm{O}(51)-\mathrm{H}(51 \mathrm{~B})$ & $0.820(18)$ \\
\hline $\mathrm{C}(32)-\mathrm{H}(32 \mathrm{~A})$ & 0.9500 & & \\
\hline C(33)-C(37) & $1.546(2)$ & $\mathrm{O}(2)-\mathrm{C}(1)-\mathrm{O}(1)$ & $124.55(12)$ \\
\hline C(33)-C(34) & $1.5522(19)$ & $\mathrm{O}(2)-\mathrm{C}(1)-\mathrm{C}(2)$ & $117.70(11)$ \\
\hline C(33)-H(33) & 1.0000 & $\mathrm{O}(1)-\mathrm{C}(1)-\mathrm{C}(2)$ & $117.75(11)$ \\
\hline $\mathrm{C}(34)-\mathrm{H}(34 \mathrm{~A})$ & 0.9900 & $\mathrm{C}(3)-\mathrm{C}(2)-\mathrm{C}(1)$ & $108.09(10)$ \\
\hline $\mathrm{C}(34)-\mathrm{H}(34 \mathrm{~B})$ & 0.9900 & $C(3)-C(2)-C(8)$ & $113.83(11)$ \\
\hline$C(35)-C(36)$ & $1.5391(19)$ & $\mathrm{C}(1)-\mathrm{C}(2)-\mathrm{C}(8)$ & $111.97(10)$ \\
\hline $\mathrm{C}(35)-\mathrm{H}(35 \mathrm{~A})$ & 0.9900 & $\mathrm{C}(3)-\mathrm{C}(2)-\mathrm{H}(2)$ & 107.6 \\
\hline $\mathrm{C}(35)-\mathrm{H}(35 \mathrm{~B})$ & 0.9900 & $\mathrm{C}(1)-\mathrm{C}(2)-\mathrm{H}(2)$ & 107.6 \\
\hline C(36)-C(37) & $1.5315(18)$ & $\mathrm{C}(8)-\mathrm{C}(2)-\mathrm{H}(2)$ & 107.6 \\
\hline $\mathrm{C}(36)-\mathrm{H}(36 \mathrm{~A})$ & 0.9900 & $C(4)-C(3)-C(2)$ & $117.95(11)$ \\
\hline $\mathrm{C}(36)-\mathrm{H}(36 \mathrm{~B})$ & 0.9900 & $\mathrm{C}(4)-\mathrm{C}(3)-\mathrm{H}(3 \mathrm{~A})$ & 107.8 \\
\hline $\mathrm{C}(37)-\mathrm{C}(38)$ & $1.5332(18)$ & $\mathrm{C}(2)-\mathrm{C}(3)-\mathrm{H}(3 \mathrm{~A})$ & 107.8 \\
\hline $\mathrm{C}(37)-\mathrm{H}(37)$ & 1.0000 & $\mathrm{C}(4)-\mathrm{C}(3)-\mathrm{H}(3 \mathrm{AB})$ & 107.8 \\
\hline C(38)-C(39) & $1.5442(18)$ & $\mathrm{C}(2)-\mathrm{C}(3)-\mathrm{H}(3 \mathrm{AB})$ & 107.8 \\
\hline $\mathrm{C}(38)-\mathrm{H}(38 \mathrm{~A})$ & 0.9900 & $\mathrm{H}(3 \mathrm{~A})-\mathrm{C}(3)-\mathrm{H}(3 \mathrm{AB})$ & 107.2 \\
\hline $\mathrm{C}(38)-\mathrm{H}(38 \mathrm{~B})$ & 0.9900 & $C(5)-C(4)-C(3)$ & $113.51(12)$ \\
\hline
\end{tabular}




\begin{tabular}{|c|c|c|c|}
\hline $\mathrm{C}(5)-\mathrm{C}(4)-\mathrm{H}(4 \mathrm{~A})$ & 108.9 & $\mathrm{C}(23)-\mathrm{N}(12)-\mathrm{C}(24)$ & $116.72(12)$ \\
\hline $\mathrm{C}(3)-\mathrm{C}(4)-\mathrm{H}(4 \mathrm{~A})$ & 108.9 & $\mathrm{C}(12)-\mathrm{C}(11)-\mathrm{H}(11 \mathrm{~A})$ & 120.0 \\
\hline $\mathrm{C}(5)-\mathrm{C}(4)-\mathrm{H}(4 \mathrm{AB})$ & 108.9 & $\mathrm{C}(12)-\mathrm{C}(11)-\mathrm{H}(11 \mathrm{~B})$ & 120.0 \\
\hline $\mathrm{C}(3)-\mathrm{C}(4)-\mathrm{H}(4 \mathrm{AB})$ & 108.9 & $\mathrm{H}(11 \mathrm{~A})-\mathrm{C}(11)-\mathrm{H}(11 \mathrm{~B})$ & 120.0 \\
\hline $\mathrm{H}(4 \mathrm{~A})-\mathrm{C}(4)-\mathrm{H}(4 \mathrm{AB})$ & 107.7 & $\mathrm{C}(12)-\mathrm{C}(11 \mathrm{~A})-\mathrm{H}(11 \mathrm{C})$ & 120.0 \\
\hline$C(4)-C(5)-C(6)$ & $115.34(11)$ & $C(12)-C(11 A)-H(11 D)$ & 120.0 \\
\hline $\mathrm{C}(4)-\mathrm{C}(5)-\mathrm{H}(5 \mathrm{~A})$ & 108.4 & $\mathrm{H}(11 \mathrm{C})-\mathrm{C}(11 \mathrm{~A})-\mathrm{H}(11 \mathrm{D})$ & 120.0 \\
\hline$C(6)-C(5)-H(5 A)$ & 108.4 & $\mathrm{C}(11 \mathrm{~A})-\mathrm{C}(12)-\mathrm{C}(13)$ & $131.9(9)$ \\
\hline $\mathrm{C}(4)-\mathrm{C}(5)-\mathrm{H}(5 \mathrm{AB})$ & 108.4 & $\mathrm{C}(11)-\mathrm{C}(12)-\mathrm{C}(13)$ & $121.1(4)$ \\
\hline $\mathrm{C}(6)-\mathrm{C}(5)-\mathrm{H}(5 \mathrm{AB})$ & 108.4 & $\mathrm{C}(11)-\mathrm{C}(12)-\mathrm{H}(12)$ & 119.5 \\
\hline $\mathrm{H}(5 \mathrm{~A})-\mathrm{C}(5)-\mathrm{H}(5 \mathrm{AB})$ & 107.5 & $\mathrm{C}(13)-\mathrm{C}(12)-\mathrm{H}(12)$ & 119.5 \\
\hline$C(5)-C(6)-C(7)$ & $116.12(12)$ & $\mathrm{C}(11 \mathrm{~A})-\mathrm{C}(12)-\mathrm{H}(12 \mathrm{~A})$ & 114.1 \\
\hline $\mathrm{C}(5)-\mathrm{C}(6)-\mathrm{H}(6 \mathrm{~A})$ & 108.3 & $\mathrm{C}(13)-\mathrm{C}(12)-\mathrm{H}(12 \mathrm{~A})$ & 114.1 \\
\hline$C(7)-C(6)-H(6 A)$ & 108.3 & $C(12)-C(13)-C(17)$ & $113.05(12)$ \\
\hline $\mathrm{C}(5)-\mathrm{C}(6)-\mathrm{H}(6 \mathrm{AB})$ & 108.3 & $C(12)-C(13)-C(14)$ & $110.62(12)$ \\
\hline$C(7)-C(6)-H(6 A B)$ & 108.3 & $C(17)-C(13)-C(14)$ & $108.54(11)$ \\
\hline $\mathrm{H}(6 \mathrm{~A})-\mathrm{C}(6)-\mathrm{H}(6 \mathrm{AB})$ & 107.4 & $\mathrm{C}(12)-\mathrm{C}(13)-\mathrm{H}(13)$ & 108.2 \\
\hline$C(6)-C(7)-C(8)$ & $115.63(11)$ & $\mathrm{C}(17)-\mathrm{C}(13)-\mathrm{H}(13)$ & 108.2 \\
\hline $\mathrm{C}(6)-\mathrm{C}(7)-\mathrm{H}(7 \mathrm{~A})$ & 108.4 & $\mathrm{C}(14)-\mathrm{C}(13)-\mathrm{H}(13)$ & 108.2 \\
\hline$C(8)-C(7)-H(7 A)$ & 108.4 & $\mathrm{~N}(11)-\mathrm{C}(14)-\mathrm{C}(13)$ & $109.30(11)$ \\
\hline $\mathrm{C}(6)-\mathrm{C}(7)-\mathrm{H}(7 \mathrm{AB})$ & 108.4 & $\mathrm{~N}(11)-\mathrm{C}(14)-\mathrm{H}(14 \mathrm{~A})$ & 109.8 \\
\hline $\mathrm{C}(8)-\mathrm{C}(7)-\mathrm{H}(7 \mathrm{AB})$ & 108.4 & $\mathrm{C}(13)-\mathrm{C}(14)-\mathrm{H}(14 \mathrm{~A})$ & 109.8 \\
\hline $\mathrm{H}(7 \mathrm{~A})-\mathrm{C}(7)-\mathrm{H}(7 \mathrm{AB})$ & 107.4 & $\mathrm{~N}(11)-\mathrm{C}(14)-\mathrm{H}(14 \mathrm{~B})$ & 109.8 \\
\hline $\mathrm{C}(7)-\mathrm{C}(8)-\mathrm{C}(9)$ & $112.23(11)$ & $\mathrm{C}(13)-\mathrm{C}(14)-\mathrm{H}(14 \mathrm{~B})$ & 109.8 \\
\hline $\mathrm{C}(7)-\mathrm{C}(8)-\mathrm{C}(2)$ & $110.19(11)$ & $\mathrm{H}(14 \mathrm{~A})-\mathrm{C}(14)-\mathrm{H}(14 \mathrm{~B})$ & 108.3 \\
\hline $\mathrm{C}(9)-\mathrm{C}(8)-\mathrm{C}(2)$ & $108.72(10)$ & $\mathrm{N}(11)-\mathrm{C}(15)-\mathrm{C}(16)$ & $109.53(11)$ \\
\hline $\mathrm{C}(7)-\mathrm{C}(8)-\mathrm{H}(8)$ & 108.5 & $\mathrm{~N}(11)-\mathrm{C}(15)-\mathrm{H}(15 \mathrm{~A})$ & 109.8 \\
\hline $\mathrm{C}(9)-\mathrm{C}(8)-\mathrm{H}(8)$ & 108.5 & $\mathrm{C}(16)-\mathrm{C}(15)-\mathrm{H}(15 \mathrm{~A})$ & 109.8 \\
\hline $\mathrm{C}(2)-\mathrm{C}(8)-\mathrm{H}(8)$ & 108.5 & $\mathrm{~N}(11)-\mathrm{C}(15)-\mathrm{H}(15 \mathrm{~B})$ & 109.8 \\
\hline $\mathrm{O}(3)-\mathrm{C}(9)-\mathrm{O}(4)$ & $124.82(12)$ & $\mathrm{C}(16)-\mathrm{C}(15)-\mathrm{H}(15 \mathrm{~B})$ & 109.8 \\
\hline $\mathrm{O}(3)-\mathrm{C}(9)-\mathrm{C}(8)$ & $117.44(11)$ & $\mathrm{H}(15 \mathrm{~A})-\mathrm{C}(15)-\mathrm{H}(15 \mathrm{~B})$ & 108.2 \\
\hline $\mathrm{O}(4)-\mathrm{C}(9)-\mathrm{C}(8)$ & $117.69(11)$ & $C(17)-C(16)-C(15)$ & $108.77(11)$ \\
\hline $\mathrm{C}(20)-\mathrm{O}(11)-\mathrm{H}(11 \mathrm{O})$ & $106.6(14)$ & $\mathrm{C}(17)-\mathrm{C}(16)-\mathrm{H}(16 \mathrm{~A})$ & 109.9 \\
\hline $\mathrm{C}(27)-\mathrm{O}(12)-\mathrm{C}(30)$ & $117.43(11)$ & $\mathrm{C}(15)-\mathrm{C}(16)-\mathrm{H}(16 \mathrm{~A})$ & 109.9 \\
\hline $\mathrm{C}(14)-\mathrm{N}(11)-\mathrm{C}(15)$ & $108.59(10)$ & $\mathrm{C}(17)-\mathrm{C}(16)-\mathrm{H}(16 \mathrm{~B})$ & 109.9 \\
\hline C(14)-N(11)-C(19) & 107.71(10) & $\mathrm{C}(15)-\mathrm{C}(16)-\mathrm{H}(16 \mathrm{~B})$ & 109.9 \\
\hline$C(15)-N(11)-C(19)$ & $114.22(11)$ & $\mathrm{H}(16 \mathrm{~A})-\mathrm{C}(16)-\mathrm{H}(16 \mathrm{~B})$ & 108.3 \\
\hline $\mathrm{C}(14)-\mathrm{N}(11)-\mathrm{H}(11)$ & $114.5(12)$ & $C(16)-C(17)-C(18)$ & $108.00(11)$ \\
\hline $\mathrm{C}(15)-\mathrm{N}(11)-\mathrm{H}(11)$ & $102.3(11)$ & $C(16)-C(17)-C(13)$ & $108.62(11)$ \\
\hline $\mathrm{C}(19)-\mathrm{N}(11)-\mathrm{H}(11)$ & $109.7(11)$ & $C(18)-C(17)-C(13)$ & $110.60(12)$ \\
\hline
\end{tabular}




\begin{tabular}{|c|c|c|c|}
\hline $\mathrm{C}(16)-\mathrm{C}(17)-\mathrm{H}(17)$ & 109.9 & $\mathrm{O}(12)-\mathrm{C}(27)-\mathrm{C}(26)$ & $113.53(13)$ \\
\hline $\mathrm{C}(18)-\mathrm{C}(17)-\mathrm{H}(17)$ & 109.9 & $C(28)-C(27)-C(26)$ & $120.68(13)$ \\
\hline $\mathrm{C}(13)-\mathrm{C}(17)-\mathrm{H}(17)$ & 109.9 & $\mathrm{C}(27)-\mathrm{C}(28)-\mathrm{C}(29)$ & $119.99(12)$ \\
\hline C(17)-C(18)-C(19) & $109.67(11)$ & $\mathrm{C}(27)-\mathrm{C}(28)-\mathrm{H}(28)$ & 120.0 \\
\hline $\mathrm{C}(17)-\mathrm{C}(18)-\mathrm{H}(18 \mathrm{~A})$ & 109.7 & $\mathrm{C}(29)-\mathrm{C}(28)-\mathrm{H}(28)$ & 120.0 \\
\hline $\mathrm{C}(19)-\mathrm{C}(18)-\mathrm{H}(18 \mathrm{~A})$ & 109.7 & $\mathrm{C}(24)-\mathrm{C}(29)-\mathrm{C}(28)$ & $119.24(13)$ \\
\hline $\mathrm{C}(17)-\mathrm{C}(18)-\mathrm{H}(18 \mathrm{~B})$ & 109.7 & $C(24)-C(29)-C(21)$ & $116.90(13)$ \\
\hline $\mathrm{C}(19)-\mathrm{C}(18)-\mathrm{H}(18 \mathrm{~B})$ & 109.7 & $C(28)-C(29)-C(21)$ & $123.85(12)$ \\
\hline $\mathrm{H}(18 \mathrm{~A})-\mathrm{C}(18)-\mathrm{H}(18 \mathrm{~B})$ & 108.2 & $\mathrm{O}(12)-\mathrm{C}(30)-\mathrm{H}(30 \mathrm{~A})$ & 109.5 \\
\hline $\mathrm{N}(11)-\mathrm{C}(19)-\mathrm{C}(18)$ & $108.72(10)$ & $\mathrm{O}(12)-\mathrm{C}(30)-\mathrm{H}(30 \mathrm{~B})$ & 109.5 \\
\hline $\mathrm{N}(11)-\mathrm{C}(19)-\mathrm{C}(20)$ & $113.00(10)$ & $\mathrm{H}(30 \mathrm{~A})-\mathrm{C}(30)-\mathrm{H}(30 \mathrm{~B})$ & 109.5 \\
\hline$C(18)-C(19)-C(20)$ & $113.95(11)$ & $\mathrm{O}(12)-\mathrm{C}(30)-\mathrm{H}(30 \mathrm{C})$ & 109.5 \\
\hline N(11)-C(19)-H(19) & 106.9 & $\mathrm{H}(30 \mathrm{~A})-\mathrm{C}(30)-\mathrm{H}(30 \mathrm{C})$ & 109.5 \\
\hline C(18)-C(19)-H(19) & 106.9 & $\mathrm{H}(30 \mathrm{~B})-\mathrm{C}(30)-\mathrm{H}(30 \mathrm{C})$ & 109.5 \\
\hline $\mathrm{C}(20)-\mathrm{C}(19)-\mathrm{H}(19)$ & 106.9 & $\mathrm{C}(40)-\mathrm{O}(31)-\mathrm{H}(31 \mathrm{O})$ & $108.6(14)$ \\
\hline $\mathrm{O}(11)-\mathrm{C}(20)-\mathrm{C}(21)$ & $110.74(11)$ & $\mathrm{C}(47)-\mathrm{O}(32)-\mathrm{C}(50)$ & $117.28(11)$ \\
\hline $\mathrm{O}(11)-\mathrm{C}(20)-\mathrm{C}(19)$ & $111.57(11)$ & $\mathrm{C}(34)-\mathrm{N}(31)-\mathrm{C}(35)$ & $108.27(10)$ \\
\hline $\mathrm{C}(21)-\mathrm{C}(20)-\mathrm{C}(19)$ & $106.48(10)$ & $\mathrm{C}(34)-\mathrm{N}(31)-\mathrm{C}(39)$ & $107.44(10)$ \\
\hline $\mathrm{O}(11)-\mathrm{C}(20)-\mathrm{H}(20)$ & 109.3 & $\mathrm{C}(35)-\mathrm{N}(31)-\mathrm{C}(39)$ & $114.60(10)$ \\
\hline $\mathrm{C}(21)-\mathrm{C}(20)-\mathrm{H}(20)$ & 109.3 & $\mathrm{C}(34)-\mathrm{N}(31)-\mathrm{H}(31)$ & $114.1(12)$ \\
\hline $\mathrm{C}(19)-\mathrm{C}(20)-\mathrm{H}(20)$ & 109.3 & $\mathrm{C}(35)-\mathrm{N}(31)-\mathrm{H}(31)$ & $104.0(12)$ \\
\hline $\mathrm{C}(22)-\mathrm{C}(21)-\mathrm{C}(29)$ & $118.50(12)$ & $\mathrm{C}(39)-\mathrm{N}(31)-\mathrm{H}(31)$ & $108.6(11)$ \\
\hline$C(22)-C(21)-C(20)$ & $120.43(12)$ & $\mathrm{C}(43)-\mathrm{N}(32)-\mathrm{C}(44)$ & $116.72(12)$ \\
\hline $\mathrm{C}(29)-\mathrm{C}(21)-\mathrm{C}(20)$ & $120.98(12)$ & $\mathrm{C}(32)-\mathrm{C}(31)-\mathrm{H}(31 \mathrm{~A})$ & 120.0 \\
\hline $\mathrm{C}(21)-\mathrm{C}(22)-\mathrm{C}(23)$ & 119.66(13) & $\mathrm{C}(32)-\mathrm{C}(31)-\mathrm{H}(31 \mathrm{~B})$ & 120.0 \\
\hline $\mathrm{C}(21)-\mathrm{C}(22)-\mathrm{H}(22)$ & 120.2 & $\mathrm{H}(31 \mathrm{~A})-\mathrm{C}(31)-\mathrm{H}(31 \mathrm{~B})$ & 120.0 \\
\hline $\mathrm{C}(23)-\mathrm{C}(22)-\mathrm{H}(22)$ & 120.2 & $\mathrm{C}(32)-\mathrm{C}(31 \mathrm{~A})-\mathrm{H}(31 \mathrm{C})$ & 120.0 \\
\hline $\mathrm{N}(12)-\mathrm{C}(23)-\mathrm{C}(22)$ & $124.38(13)$ & $\mathrm{C}(32)-\mathrm{C}(31 \mathrm{~A})-\mathrm{H}(31 \mathrm{D})$ & 120.0 \\
\hline $\mathrm{N}(12)-\mathrm{C}(23)-\mathrm{H}(23)$ & 117.8 & $\mathrm{H}(31 \mathrm{C})-\mathrm{C}(31 \mathrm{~A})-\mathrm{H}(31 \mathrm{D})$ & 120.0 \\
\hline $\mathrm{C}(22)-\mathrm{C}(23)-\mathrm{H}(23)$ & 117.8 & $\mathrm{C}(31 \mathrm{~A})-\mathrm{C}(32)-\mathrm{C}(33)$ & $129.2(12)$ \\
\hline $\mathrm{N}(12)-\mathrm{C}(24)-\mathrm{C}(25)$ & $117.40(12)$ & $\mathrm{C}(31)-\mathrm{C}(32)-\mathrm{C}(33)$ & $121.7(6)$ \\
\hline $\mathrm{N}(12)-\mathrm{C}(24)-\mathrm{C}(29)$ & $123.78(13)$ & $\mathrm{C}(31)-\mathrm{C}(32)-\mathrm{H}(32)$ & 119.1 \\
\hline$C(25)-C(24)-C(29)$ & $118.81(13)$ & $\mathrm{C}(33)-\mathrm{C}(32)-\mathrm{H}(32)$ & 119.1 \\
\hline$C(26)-C(25)-C(24)$ & $120.98(13)$ & $\mathrm{C}(31 \mathrm{~A})-\mathrm{C}(32)-\mathrm{H}(32 \mathrm{~A})$ & 115.4 \\
\hline $\mathrm{C}(26)-\mathrm{C}(25)-\mathrm{H}(25)$ & 119.5 & $\mathrm{C}(33)-\mathrm{C}(32)-\mathrm{H}(32 \mathrm{~A})$ & 115.4 \\
\hline $\mathrm{C}(24)-\mathrm{C}(25)-\mathrm{H}(25)$ & 119.5 & $\mathrm{C}(32)-\mathrm{C}(33)-\mathrm{C}(37)$ & $113.05(12)$ \\
\hline$C(25)-C(26)-C(27)$ & $120.20(13)$ & $\mathrm{C}(32)-\mathrm{C}(33)-\mathrm{C}(34)$ & $110.59(12)$ \\
\hline $\mathrm{C}(25)-\mathrm{C}(26)-\mathrm{H}(26)$ & 119.9 & $\mathrm{C}(37)-\mathrm{C}(33)-\mathrm{C}(34)$ & $108.54(11)$ \\
\hline $\mathrm{C}(27)-\mathrm{C}(26)-\mathrm{H}(26)$ & 119.9 & $\mathrm{C}(32)-\mathrm{C}(33)-\mathrm{H}(33)$ & 108.2 \\
\hline $\mathrm{O}(12)-\mathrm{C}(27)-\mathrm{C}(28)$ & $125.78(13)$ & $\mathrm{C}(37)-\mathrm{C}(33)-\mathrm{H}(33)$ & 108.2 \\
\hline
\end{tabular}




\begin{tabular}{|c|c|c|c|}
\hline $\mathrm{C}(34)-\mathrm{C}(33)-\mathrm{H}(33)$ & 108.2 & $\mathrm{O}(31)-\mathrm{C}(40)-\mathrm{H}(40)$ & 109.3 \\
\hline $\mathrm{N}(31)-\mathrm{C}(34)-\mathrm{C}(33)$ & $109.13(11)$ & $\mathrm{C}(41)-\mathrm{C}(40)-\mathrm{H}(40)$ & 109.3 \\
\hline $\mathrm{N}(31)-\mathrm{C}(34)-\mathrm{H}(34 \mathrm{~A})$ & 109.9 & $\mathrm{C}(39)-\mathrm{C}(40)-\mathrm{H}(40)$ & 109.3 \\
\hline $\mathrm{C}(33)-\mathrm{C}(34)-\mathrm{H}(34 \mathrm{~A})$ & 109.9 & $C(42)-C(41)-C(49)$ & $118.44(12)$ \\
\hline $\mathrm{N}(31)-\mathrm{C}(34)-\mathrm{H}(34 \mathrm{~B})$ & 109.9 & $\mathrm{C}(42)-\mathrm{C}(41)-\mathrm{C}(40)$ & $120.90(12)$ \\
\hline $\mathrm{C}(33)-\mathrm{C}(34)-\mathrm{H}(34 \mathrm{~B})$ & 109.9 & $C(49)-C(41)-C(40)$ & $120.58(12)$ \\
\hline $\mathrm{H}(34 \mathrm{~A})-\mathrm{C}(34)-\mathrm{H}(34 \mathrm{~B})$ & 108.3 & $\mathrm{C}(41)-\mathrm{C}(42)-\mathrm{C}(43)$ & $119.54(13)$ \\
\hline $\mathrm{N}(31)-\mathrm{C}(35)-\mathrm{C}(36)$ & $109.35(10)$ & $\mathrm{C}(41)-\mathrm{C}(42)-\mathrm{H}(42)$ & 120.2 \\
\hline $\mathrm{N}(31)-\mathrm{C}(35)-\mathrm{H}(35 \mathrm{~A})$ & 109.8 & $\mathrm{C}(43)-\mathrm{C}(42)-\mathrm{H}(42)$ & 120.2 \\
\hline $\mathrm{C}(36)-\mathrm{C}(35)-\mathrm{H}(35 \mathrm{~A})$ & 109.8 & $\mathrm{~N}(32)-\mathrm{C}(43)-\mathrm{C}(42)$ & $124.45(13)$ \\
\hline $\mathrm{N}(31)-\mathrm{C}(35)-\mathrm{H}(35 \mathrm{~B})$ & 109.8 & $\mathrm{~N}(32)-\mathrm{C}(43)-\mathrm{H}(43)$ & 117.8 \\
\hline $\mathrm{C}(36)-\mathrm{C}(35)-\mathrm{H}(35 \mathrm{~B})$ & 109.8 & $\mathrm{C}(42)-\mathrm{C}(43)-\mathrm{H}(43)$ & 117.8 \\
\hline $\mathrm{H}(35 \mathrm{~A})-\mathrm{C}(35)-\mathrm{H}(35 \mathrm{~B})$ & 108.3 & $\mathrm{~N}(32)-\mathrm{C}(44)-\mathrm{C}(49)$ & $123.68(13)$ \\
\hline $\mathrm{C}(37)-\mathrm{C}(36)-\mathrm{C}(35)$ & $108.71(11)$ & N(32)-C(44)-C(45) & $117.57(12)$ \\
\hline $\mathrm{C}(37)-\mathrm{C}(36)-\mathrm{H}(36 \mathrm{~A})$ & 109.9 & $C(49)-C(44)-C(45)$ & $118.75(13)$ \\
\hline $\mathrm{C}(35)-\mathrm{C}(36)-\mathrm{H}(36 \mathrm{~A})$ & 109.9 & $\mathrm{C}(46)-\mathrm{C}(45)-\mathrm{C}(44)$ & $121.15(12)$ \\
\hline $\mathrm{C}(37)-\mathrm{C}(36)-\mathrm{H}(36 \mathrm{~B})$ & 109.9 & $\mathrm{C}(46)-\mathrm{C}(45)-\mathrm{H}(45)$ & 119.4 \\
\hline $\mathrm{C}(35)-\mathrm{C}(36)-\mathrm{H}(36 \mathrm{~B})$ & 109.9 & $\mathrm{C}(44)-\mathrm{C}(45)-\mathrm{H}(45)$ & 119.4 \\
\hline $\mathrm{H}(36 \mathrm{~A})-\mathrm{C}(36)-\mathrm{H}(36 \mathrm{~B})$ & 108.3 & $C(45)-C(46)-C(47)$ & $119.96(13)$ \\
\hline $\mathrm{C}(36)-\mathrm{C}(37)-\mathrm{C}(38)$ & $107.95(11)$ & $\mathrm{C}(45)-\mathrm{C}(46)-\mathrm{H}(46)$ & 120.0 \\
\hline$C(36)-C(37)-C(33)$ & $108.28(11)$ & $\mathrm{C}(47)-\mathrm{C}(46)-\mathrm{H}(46)$ & 120.0 \\
\hline $\mathrm{C}(38)-\mathrm{C}(37)-\mathrm{C}(33)$ & $110.84(11)$ & $\mathrm{O}(32)-\mathrm{C}(47)-\mathrm{C}(48)$ & $125.30(12)$ \\
\hline $\mathrm{C}(36)-\mathrm{C}(37)-\mathrm{H}(37)$ & 109.9 & $\mathrm{O}(32)-\mathrm{C}(47)-\mathrm{C}(46)$ & $113.78(12)$ \\
\hline $\mathrm{C}(38)-\mathrm{C}(37)-\mathrm{H}(37)$ & 109.9 & $\mathrm{C}(48)-\mathrm{C}(47)-\mathrm{C}(46)$ & $120.92(13)$ \\
\hline $\mathrm{C}(33)-\mathrm{C}(37)-\mathrm{H}(37)$ & 109.9 & $\mathrm{C}(47)-\mathrm{C}(48)-\mathrm{C}(49)$ & $119.77(12)$ \\
\hline $\mathrm{C}(37)-\mathrm{C}(38)-\mathrm{C}(39)$ & $109.78(11)$ & $\mathrm{C}(47)-\mathrm{C}(48)-\mathrm{H}(48)$ & 120.1 \\
\hline $\mathrm{C}(37)-\mathrm{C}(38)-\mathrm{H}(38 \mathrm{~A})$ & 109.7 & $\mathrm{C}(49)-\mathrm{C}(48)-\mathrm{H}(48)$ & 120.1 \\
\hline $\mathrm{C}(39)-\mathrm{C}(38)-\mathrm{H}(38 \mathrm{~A})$ & 109.7 & $\mathrm{C}(44)-\mathrm{C}(49)-\mathrm{C}(48)$ & $119.35(12)$ \\
\hline $\mathrm{C}(37)-\mathrm{C}(38)-\mathrm{H}(38 \mathrm{~B})$ & 109.7 & $\mathrm{C}(44)-\mathrm{C}(49)-\mathrm{C}(41)$ & $117.06(12)$ \\
\hline $\mathrm{C}(39)-\mathrm{C}(38)-\mathrm{H}(38 \mathrm{~B})$ & 109.7 & $\mathrm{C}(48)-\mathrm{C}(49)-\mathrm{C}(41)$ & $123.59(12)$ \\
\hline $\mathrm{H}(38 \mathrm{~A})-\mathrm{C}(38)-\mathrm{H}(38 \mathrm{~B})$ & 108.2 & $\mathrm{O}(32)-\mathrm{C}(50)-\mathrm{H}(50 \mathrm{~A})$ & 109.5 \\
\hline N(31)-C(39)-C(38) & $108.66(10)$ & $\mathrm{O}(32)-\mathrm{C}(50)-\mathrm{H}(50 \mathrm{~B})$ & 109.5 \\
\hline $\mathrm{N}(31)-\mathrm{C}(39)-\mathrm{C}(40)$ & $113.27(10)$ & $\mathrm{H}(50 \mathrm{~A})-\mathrm{C}(50)-\mathrm{H}(50 \mathrm{~B})$ & 109.5 \\
\hline $\mathrm{C}(38)-\mathrm{C}(39)-\mathrm{C}(40)$ & $114.34(11)$ & $\mathrm{O}(32)-\mathrm{C}(50)-\mathrm{H}(50 \mathrm{C})$ & 109.5 \\
\hline N(31)-C(39)-H(39) & 106.7 & $\mathrm{H}(50 \mathrm{~A})-\mathrm{C}(50)-\mathrm{H}(50 \mathrm{C})$ & 109.5 \\
\hline $\mathrm{C}(38)-\mathrm{C}(39)-\mathrm{H}(39)$ & 106.7 & $\mathrm{H}(50 \mathrm{~B})-\mathrm{C}(50)-\mathrm{H}(50 \mathrm{C})$ & 109.5 \\
\hline $\mathrm{C}(40)-\mathrm{C}(39)-\mathrm{H}(39)$ & 106.7 & $\mathrm{H}(51 \mathrm{~A})-\mathrm{O}(51)-\mathrm{H}(51 \mathrm{~B})$ & $106(2)$ \\
\hline $\mathrm{O}(31)-\mathrm{C}(40)-\mathrm{C}(41)$ & $110.46(11)$ & & \\
\hline $\mathrm{O}(31)-\mathrm{C}(40)-\mathrm{C}(39)$ & $112.09(11)$ & & \\
\hline $\mathrm{C}(41)-\mathrm{C}(40)-\mathrm{C}(39)$ & $106.17(10)$ & & \\
\hline
\end{tabular}


Table S4. Anisotropic displacement parameters $\left(\AA^{2} \times 10^{3}\right)$ for $(-)-5$. The anisotropic displacement factor exponent takes the form: $-2 \pi^{2}\left[h^{2} a^{* 2} U^{11}+\ldots+2 h k a^{*} b^{*} U^{12}\right]$

\begin{tabular}{|c|c|c|c|c|c|c|}
\hline & $\mathrm{U}^{11}$ & $\mathrm{U}^{22}$ & $\mathrm{U}^{33}$ & $\mathrm{U}^{23}$ & $\mathrm{U}^{13}$ & $\mathrm{U}^{12}$ \\
\hline $\mathrm{O}(1)$ & $17(1)$ & $16(1)$ & $17(1)$ & $-2(1)$ & $0(1)$ & $2(1)$ \\
\hline $\mathrm{O}(2)$ & $17(1)$ & $13(1)$ & $17(1)$ & $0(1)$ & $-1(1)$ & $-2(1)$ \\
\hline $\mathrm{O}(3)$ & $22(1)$ & $16(1)$ & $19(1)$ & $-2(1)$ & $3(1)$ & $-5(1)$ \\
\hline $\mathrm{O}(4)$ & $16(1)$ & $12(1)$ & $17(1)$ & $-1(1)$ & $-1(1)$ & $1(1)$ \\
\hline $\mathrm{C}(1)$ & $16(1)$ & $14(1)$ & $10(1)$ & $1(1)$ & $3(1)$ & $-1(1)$ \\
\hline$C(2)$ & $16(1)$ & $12(1)$ & $14(1)$ & $1(1)$ & $0(1)$ & $-2(1)$ \\
\hline$C(3)$ & $18(1)$ & $24(1)$ & $14(1)$ & $-1(1)$ & $2(1)$ & $-2(1)$ \\
\hline$C(4)$ & $17(1)$ & $18(1)$ & $22(1)$ & $-1(1)$ & $4(1)$ & $-1(1)$ \\
\hline$C(5)$ & $18(1)$ & $22(1)$ & $27(1)$ & $-3(1)$ & $0(1)$ & $4(1)$ \\
\hline$C(6)$ & $18(1)$ & $30(1)$ & $24(1)$ & $-4(1)$ & $-6(1)$ & $3(1)$ \\
\hline$C(7)$ & $18(1)$ & $18(1)$ & $15(1)$ & $-2(1)$ & $-2(1)$ & $-1(1)$ \\
\hline$C(8)$ & $16(1)$ & $12(1)$ & $14(1)$ & $0(1)$ & $-2(1)$ & $1(1)$ \\
\hline $\mathrm{C}(9)$ & $15(1)$ & $14(1)$ & $10(1)$ & $1(1)$ & $-2(1)$ & $1(1)$ \\
\hline $\mathrm{O}(11)$ & $20(1)$ & $13(1)$ & $16(1)$ & $-2(1)$ & $-1(1)$ & $0(1)$ \\
\hline $\mathrm{O}(12)$ & $19(1)$ & $20(1)$ & $19(1)$ & $-1(1)$ & $2(1)$ & $1(1)$ \\
\hline $\mathrm{N}(11)$ & $13(1)$ & $11(1)$ & $16(1)$ & $1(1)$ & $-2(1)$ & $-1(1)$ \\
\hline $\mathrm{N}(12)$ & $16(1)$ & $14(1)$ & $22(1)$ & $0(1)$ & $-5(1)$ & $0(1)$ \\
\hline $\mathrm{C}(11)$ & $26(1)$ & $47(4)$ & $51(4)$ & $-28(3)$ & $-8(2)$ & $-3(2)$ \\
\hline$C(11 A)$ & $26(1)$ & $47(4)$ & $51(4)$ & $-28(3)$ & $-8(2)$ & $-3(2)$ \\
\hline$C(12)$ & $22(1)$ & $25(1)$ & $28(1)$ & $-12(1)$ & $1(1)$ & $-4(1)$ \\
\hline$C(13)$ & $12(1)$ & $18(1)$ & $20(1)$ & $-4(1)$ & $-3(1)$ & $3(1)$ \\
\hline$C(14)$ & $17(1)$ & $19(1)$ & $16(1)$ & $0(1)$ & $-4(1)$ & $1(1)$ \\
\hline$C(15)$ & $17(1)$ & $12(1)$ & $20(1)$ & $-3(1)$ & $-3(1)$ & $1(1)$ \\
\hline$C(16)$ & $17(1)$ & $19(1)$ & $16(1)$ & $-3(1)$ & $1(1)$ & $1(1)$ \\
\hline$C(17)$ & $12(1)$ & $15(1)$ & $20(1)$ & $-2(1)$ & $-1(1)$ & $0(1)$ \\
\hline$C(18)$ & $13(1)$ & $13(1)$ & $23(1)$ & $2(1)$ & $-2(1)$ & $-2(1)$ \\
\hline$C(19)$ & $12(1)$ & $10(1)$ & $16(1)$ & $0(1)$ & $-2(1)$ & $0(1)$ \\
\hline$C(20)$ & $12(1)$ & $12(1)$ & $15(1)$ & $0(1)$ & $-1(1)$ & $0(1)$ \\
\hline $\mathrm{C}(21)$ & $11(1)$ & $12(1)$ & $18(1)$ & $-2(1)$ & $-4(1)$ & $-1(1)$ \\
\hline $\mathrm{C}(22)$ & $14(1)$ & $15(1)$ & $17(1)$ & $0(1)$ & $-2(1)$ & $-1(1)$ \\
\hline $\mathrm{C}(23)$ & $15(1)$ & $15(1)$ & $20(1)$ & $3(1)$ & $-4(1)$ & $-1(1)$ \\
\hline$C(24)$ & $12(1)$ & $14(1)$ & $20(1)$ & $-2(1)$ & $-4(1)$ & $-1(1)$ \\
\hline
\end{tabular}




$\begin{array}{lllllll}\mathrm{C}(25) & 16(1) & 14(1) & 23(1) & -4(1) & -5(1) & 2(1) \\ \mathrm{C}(26) & 15(1) & 20(1) & 20(1) & -6(1) & -3(1) & 2(1) \\ \mathrm{C}(27) & 12(1) & 20(1) & 16(1) & -2(1) & -3(1) & -3(1) \\ \mathrm{C}(28) & 12(1) & 14(1) & 19(1) & -1(1) & -4(1) & 1(1) \\ \mathrm{C}(29) & 11(1) & 14(1) & 18(1) & -2(1) & -4(1) & -1(1) \\ \mathrm{C}(30) & 24(1) & 20(1) & 21(1) & 1(1) & 4(1) & -2(1) \\ \mathrm{O}(31) & 19(1) & 13(1) & 13(1) & -2(1) & -1(1) & -1(1) \\ \mathrm{O}(32) & 18(1) & 19(1) & 17(1) & 2(1) & -3(1) & -2(1) \\ \mathrm{N}(31) & 12(1) & 12(1) & 13(1) & 1(1) & 1(1) & 0(1) \\ \mathrm{N}(32) & 15(1) & 14(1) & 20(1) & 2(1) & 3(1) & 1(1) \\ \mathrm{C}(31) & 29(3) & 31(3) & 38(3) & -14(2) & 11(2) & 6(2) \\ \mathrm{C}(31 \mathrm{~A}) & 14(5) & 56(11) & 51(8) & -32(8) & -4(4) & -1(4) \\ \mathrm{C}(32) & 21(1) & 21(1) & 23(1) & -7(1) & 1(1) & 2(1) \\ \mathrm{C}(33) & 11(1) & 16(1) & 18(1) & -2(1) & 3(1) & -2(1) \\ \mathrm{C}(34) & 16(1) & 18(1) & 12(1) & -1(1) & 3(1) & -1(1) \\ \mathrm{C}(35) & 16(1) & 11(1) & 17(1) & -2(1) & 1(1) & -2(1) \\ \mathrm{C}(36) & 15(1) & 20(1) & 17(1) & -3(1) & -3(1) & -1(1) \\ \mathrm{C}(37) & 12(1) & 15(1) & 18(1) & -1(1) & 0(1) & 0(1) \\ \mathrm{C}(38) & 14(1) & 13(1) & 19(1) & 2(1) & 2(1) & 1(1) \\ \mathrm{C}(39) & 12(1) & 10(1) & 15(1) & 1(1) & 1(1) & -1(1) \\ \mathrm{C}(40) & 12(1) & 12(1) & 13(1) & 0(1) & 0(1) & 0(1) \\ \mathrm{C}(41) & 11(1) & 12(1) & 16(1) & 0(1) & 3(1) & 2(1) \\ \mathrm{C}(42) & 12(1) & 16(1) & 16(1) & 0(1) & 1(1) & 1(1) \\ \mathrm{C}(43) & 15(1) & 15(1) & 18(1) & 5(1) & 3(1) & 1(1) \\ \mathrm{C}(44) & 12(1) & 14(1) & 17(1) & 1(1) & 4(1) & 1(1) \\ \mathrm{C}(45) & 15(1) & 13(1) & 20(1) & -3(1) & 4(1) & -2(1) \\ \mathrm{C}(46) & 15(1) & 18(1) & 17(1) & -4(1) & 2(1) & -2(1) \\ \mathrm{C}(47) & 12(1) & 18(1) & 15(1) & 0(1) & 2(1) & 1(1) \\ \mathrm{C}(48) & 11(1) & 13(1) & 17(1) & 0(1) & 2(1) & 0(1) \\ \mathrm{C}(49) & 10(1) & 12(1) & 16(1) & -2(1) & 3(1) & 1(1) \\ \mathrm{C}(50) & 22(1) & 18(1) & 21(1) & 4(1) & -3(1) & 1(1) \\ \mathrm{O}(51) & 15(1) & 25(1) & 20(1) & 2(1) & 0(1) & 1(1)\end{array}$


Table S5. Hydrogen coordinates ( x 104) and isotropic displacement parameters $\left(\AA^{2} \times 10^{3}\right)$ for $(-)-5$.

\begin{tabular}{|c|c|c|c|c|}
\hline & $\mathrm{x}$ & $\mathrm{y}$ & $\mathrm{z}$ & $\mathrm{U}(\mathrm{eq})$ \\
\hline $\mathrm{H}(2)$ & 1568 & 7071 & 4164 & 17 \\
\hline $\mathrm{H}(3 \mathrm{~A})$ & 1142 & 7468 & 6780 & 22 \\
\hline $\mathrm{H}(3 \mathrm{AB})$ & 1069 & 7894 & 5438 & 22 \\
\hline $\mathrm{H}(4 \mathrm{~A})$ & 323 & 7126 & 4731 & 23 \\
\hline $\mathrm{H}(4 \mathrm{AB})$ & 79 & 7509 & 6158 & 23 \\
\hline $\mathrm{H}(5 \mathrm{~A})$ & 221 & 7993 & 3237 & 27 \\
\hline $\mathrm{H}(5 \mathrm{AB})$ & -408 & 7696 & 3359 & 27 \\
\hline $\mathrm{H}(6 \mathrm{~A})$ & 210 & 7727 & 201 & 28 \\
\hline $\mathrm{H}(6 \mathrm{AB})$ & -118 & 7277 & 852 & 28 \\
\hline $\mathrm{H}(7 \mathrm{~A})$ & 952 & 7190 & -380 & 21 \\
\hline $\mathrm{H}(7 \mathrm{AB})$ & 845 & 6974 & 1741 & 21 \\
\hline $\mathrm{H}(8)$ & 1358 & 7833 & 1728 & 17 \\
\hline $\mathrm{H}(11 \mathrm{O})$ & $2656(9)$ & $8315(5)$ & $330(30)$ & 24 \\
\hline $\mathrm{H}(11)$ & 2999(8) & $8205(5)$ & $4950(30)$ & 16 \\
\hline $\mathrm{H}(11 \mathrm{~A})$ & 5316 & 8925 & 7927 & 49 \\
\hline $\mathrm{H}(11 \mathrm{~B})$ & 5121 & 9432 & 8497 & 49 \\
\hline $\mathrm{H}(11 \mathrm{C})$ & 5424 & 9124 & 7252 & 49 \\
\hline $\mathrm{H}(11 \mathrm{D})$ & 5036 & 9561 & 8029 & 49 \\
\hline $\mathrm{H}(12)$ & 4250 & 9392 & 6439 & 30 \\
\hline $\mathrm{H}(12 \mathrm{~A})$ & 4165 & 9332 & 6744 & 30 \\
\hline $\mathrm{H}(13)$ & 4757 & 8533 & 5580 & 19 \\
\hline $\mathrm{H}(14 \mathrm{~A})$ & 3906 & 8236 & 6992 & 21 \\
\hline $\mathrm{H}(14 \mathrm{~B})$ & 3554 & 8701 & 7200 & 21 \\
\hline $\mathrm{H}(15 \mathrm{~A})$ & 3405 & 7985 & 2150 & 19 \\
\hline $\mathrm{H}(15 \mathrm{~B})$ & 3900 & 7853 & 3861 & 19 \\
\hline $\mathrm{H}(16 \mathrm{~A})$ & 4645 & 8222 & 2220 & 21 \\
\hline $\mathrm{H}(16 \mathrm{~B})$ & 4120 & 8424 & 744 & 21 \\
\hline $\mathrm{H}(17)$ & 4649 & 8991 & 2665 & 19 \\
\hline $\mathrm{H}(18 \mathrm{~A})$ & 3585 & 9117 & 1433 & 19 \\
\hline $\mathrm{H}(18 \mathrm{~B})$ & 3674 & 9362 & 3511 & 19 \\
\hline $\mathrm{H}(19)$ & 2905 & 8983 & 4844 & 15 \\
\hline $\mathrm{H}(20)$ & 2220 & 8514 & 2807 & 16 \\
\hline $\mathrm{H}(22)$ & 2578 & 9330 & -622 & 18 \\
\hline $\mathrm{H}(23)$ & 2112 & 10023 & -846 & 20 \\
\hline $\mathrm{H}(25)$ & 980 & 10240 & 4654 & 21 \\
\hline
\end{tabular}




\begin{tabular}{|c|c|c|c|}
\hline $\mathrm{H}(26)$ & 727 & 9842 & 7439 \\
\hline $\mathrm{H}(28)$ & 1739 & 8795 & 5396 \\
\hline $\mathrm{H}(30 \mathrm{~A})$ & 979 & 8445 & 7597 \\
\hline $\mathrm{H}(30 \mathrm{~B})$ & 1024 & 8534 & 9921 \\
\hline $\mathrm{H}(30 \mathrm{C})$ & 1641 & 8606 & 8552 \\
\hline $\mathrm{H}(310)$ & $2726(9)$ & $6691(5)$ & $4830(30)$ \\
\hline $\mathrm{H}(31)$ & 2973(8) & $6798(5)$ & $180(30)$ \\
\hline $\mathrm{H}(31 \mathrm{~A})$ & 5295 & 6122 & -3009 \\
\hline $\mathrm{H}(31 \mathrm{~B})$ & 5093 & 5623 & -3690 \\
\hline $\mathrm{H}(31 \mathrm{C})$ & 5365 & 6000 & -2724 \\
\hline $\mathrm{H}(31 \mathrm{D})$ & 5038 & 5536 & -3448 \\
\hline $\mathrm{H}(32)$ & 4215 & 5653 & -1708 \\
\hline $\mathrm{H}(32 \mathrm{~A})$ & 4162 & 5687 & -1842 \\
\hline $\mathrm{H}(33)$ & 4709 & 6510 & -710 \\
\hline $\mathrm{H}(34 \mathrm{~A})$ & 3828 & 6790 & -2024 \\
\hline $\mathrm{H}(34 \mathrm{~B})$ & 3487 & 6319 & -2174 \\
\hline $\mathrm{H}(35 \mathrm{~A})$ & 3420 & 7030 & 2910 \\
\hline $\mathrm{H}(35 \mathrm{~B})$ & 3894 & 7158 & 1136 \\
\hline $\mathrm{H}(36 \mathrm{~A})$ & 4649 & 6784 & 2724 \\
\hline $\mathrm{H}(36 \mathrm{~B})$ & 4133 & 6572 & 4204 \\
\hline $\mathrm{H}(37)$ & 4654 & 6022 & 2146 \\
\hline $\mathrm{H}(38 \mathrm{~A})$ & 3614 & 5878 & 3498 \\
\hline H(38B) & 3672 & 5652 & 1368 \\
\hline H(39) & 2875 & 6034 & 235 \\
\hline $\mathrm{H}(40)$ & 2217 & 6483 & 2414 \\
\hline $\mathrm{H}(42)$ & 2620 & 5670 & 5794 \\
\hline $\mathrm{H}(43)$ & 2174 & 4970 & 6026 \\
\hline $\mathrm{H}(45)$ & 1053 & 4734 & 518 \\
\hline $\mathrm{H}(46)$ & 775 & 5130 & -2248 \\
\hline $\mathrm{H}(48)$ & 1742 & 6195 & -182 \\
\hline $\mathrm{H}(50 \mathrm{~A})$ & 1654 & 6370 & -3394 \\
\hline $\mathrm{H}(50 \mathrm{~B})$ & 1008 & 6534 & -2355 \\
\hline $\mathrm{H}(50 \mathrm{C})$ & 1021 & 6448 & -4685 \\
\hline $\mathrm{H}(51 \mathrm{~A})$ & $3277(10)$ & $7452(6)$ & $-3070(30)$ \\
\hline $\mathrm{H}(51 \mathrm{~B})$ & $3186(9)$ & $7614(6)$ & $-1270(30)$ \\
\hline
\end{tabular}


Table S6. Hydrogen bonds for (-)-5 [ $\AA$ and $\left.{ }^{\circ}\right]$.

\begin{tabular}{lcccc}
\hline D-H...A & d(D-H) & $d(H \ldots A)$ & $d(D . . A)$ & $<($ DHA $)$ \\
\hline $\mathrm{O}(11)-\mathrm{H}(11 \mathrm{O}) \ldots \mathrm{O}(3)$ & $0.839(16)$ & $1.859(17)$ & $2.6932(14)$ & $173(2)$ \\
$\mathrm{N}(11)-\mathrm{H}(11) \ldots \mathrm{O}(2)$ & $0.928(15)$ & $1.732(16)$ & $2.6398(15)$ & $165.1(17)$ \\
$\mathrm{O}(31)-\mathrm{H}(31 \mathrm{O}) \ldots \mathrm{O}(1)$ & $0.852(16)$ & $1.851(17)$ & $2.7004(14)$ & $174.2(18)$ \\
$\mathrm{N}(31)-\mathrm{H}(31) \ldots \mathrm{O}(4)$ & $0.889(15)$ & $1.850(16)$ & $2.7135(15)$ & $163.4(17)$ \\
$\mathrm{O}(51)-\mathrm{H}(51 \mathrm{~A}) \ldots \mathrm{O}(1) \# 1$ & $0.847(18)$ & $1.924(18)$ & $2.7560(15)$ & $167(2)$ \\
$\mathrm{O}(51)-\mathrm{H}(51 \mathrm{~B}) \ldots \mathrm{O}(3)$ & $0.820(18)$ & $1.934(18)$ & $2.7512(15)$ & $174(2)$ \\
& & & & \\
\hline
\end{tabular}

Symmetry transformations used to generate equivalent atoms:

\#1 x,y,z-1 


\section{Optical Rotatory Dispersion Experiments}

Table S7. Measured specific rotation of $(+)-1$ and computed (Boltzmann-averaged (relative free energies computed at MP2/cc-pVTZ) over five conformers, 1-I to 1-V) specific rotation of $(R, R)-\mathbf{1}$ at different wavelengths at B3LYP/6-311++G( $2 d, 2 p)$. The values are used to generate Figure 2 .

\begin{tabular}{|l|l|l|l|l|l|l|}
\hline & $633 \mathrm{~nm}$ & $589 \mathrm{~nm}$ & $546 \mathrm{~nm}$ & $436 \mathrm{~nm}$ & $405 \mathrm{~nm}$ & $365 \mathrm{~nm}$ \\
\hline Experiment $\left(\mathrm{c} 0.525, \mathrm{CDCl}_{3}\right)$ & 11.9 & 13.1 & 15.6 & 24.3 & 28.3 & 34.5 \\
\hline B3LYP/6-311++G(2d,2p) & 9.1 & 10.5 & 12.2 & 18.5 & 21.1 & 24.9 \\
\hline
\end{tabular}

Table S8. Measured specific rotation of $(+)-\mathbf{1}$ and computed (Boltzmann-averaged (relative free energies computed at B3LYP/6-311++G(2d,2p)) over five conformers, 1-I to 1-V) specific rotation of $(R, R)-1$ at different wavelengths at $\mathrm{B} 3 \mathrm{LYP} / 6-311++\mathrm{G}(2 d, 2 p)$.

\begin{tabular}{|l|l|l|l|l|l|l|}
\hline & $633 \mathrm{~nm}$ & $589 \mathrm{~nm}$ & $546 \mathrm{~nm}$ & $436 \mathrm{~nm}$ & $405 \mathrm{~nm}$ & $365 \mathrm{~nm}$ \\
\hline Experiment $\left(\mathrm{c} 0.525, \mathrm{CDCl}_{3}\right)$ & 11.9 & 13.1 & 15.6 & 24.3 & 28.3 & 34.5 \\
\hline B3LYP/6-311++G(2d,2p) & 6.1 & 7.0 & 8.1 & 11.8 & 13.1 & 14.8 \\
\hline
\end{tabular}

Table S9. Measured specific rotation of (+)-1 and computed (Boltzmann-averaged (relative free energies computed at MP2/cc-pVTZ) over five conformers, 1-I to 1-V) specific rotation of $(R, R)-\mathbf{1}$ at different wavelengths at CAM-B3LYP/6-311++G $(2 d, 2 p)$.

\begin{tabular}{|l|l|l|l|l|l|l|}
\hline & $633 \mathrm{~nm}$ & $589 \mathrm{~nm}$ & $546 \mathrm{~nm}$ & $436 \mathrm{~nm}$ & $405 \mathrm{~nm}$ & $365 \mathrm{~nm}$ \\
\hline Experiment $\left(\mathrm{c} 0.525, \mathrm{CDCl}_{3}\right)$ & 11.9 & 13.1 & 15.6 & 24.3 & 28.3 & 34.5 \\
\hline CAM-B3LYP/6-311++G(2d,2p) & 8.1 & 9.3 & 10.8 & 16.7 & 19.1 & 23.0 \\
\hline
\end{tabular}

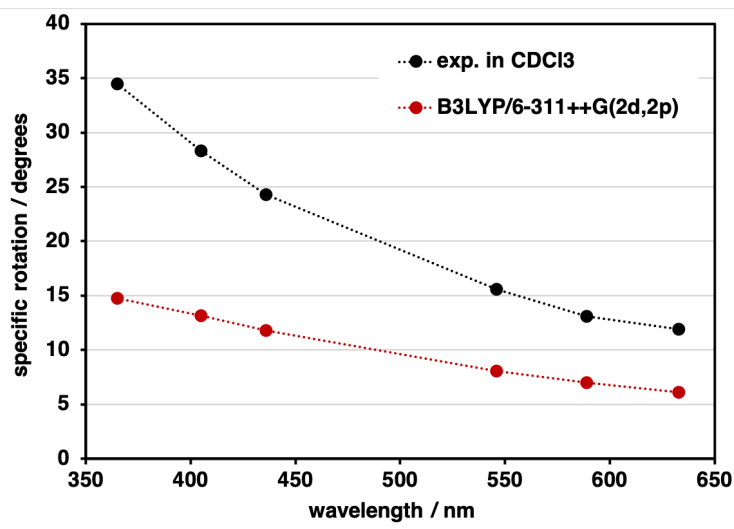

Figure S2. Measured specific rotation of $(+)-\mathbf{1}$ and Boltzmann-averaged (relative free energies at $\mathrm{B} 3 \mathrm{LYP} / 6-311++\mathrm{G}(2 d, 2 p))$ computed specific rotation of $(R, R)-\mathbf{1}$ at different wavelengths at $\mathrm{B} 3 \mathrm{LYP} / 6-311++\mathrm{G}(2 d, 2 p)$.

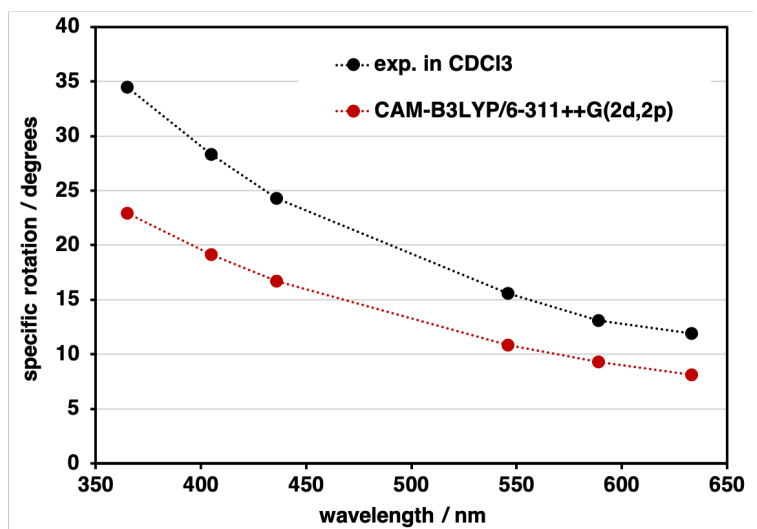

Figure S3. Measured specific rotation of $(+)-\mathbf{1}$ and Boltzmann-averaged (relative free energies at $\mathrm{MP} 2 / \mathrm{cc}-\mathrm{pVTZ}$ ) computed specific rotation of $(R, R)$ 1 at different wavelengths at CAM-B3LYP/6$311++\mathrm{G}(2 d, 2 p)$. 


\section{Vibrational Circular Dichroism Experiments}

VCD spectra were recorded with a Bruker PMA50 photoelastic modulator unit attached to a Bruker Vertex 70 spectrometer in conjunction with a Hinds PEM-100 controller and a Stanford Research Systems SR830 DSP lock-in amplifier (LIA). The LIA was set to a voltage of $1 \mathrm{mV}$ and the PEM was tuned to maximize its sensitivity in the spectral region around $1000 \mathrm{~cm}^{-1}$. The spectral resolution for IR spectra was $4 \mathrm{~cm}^{-1}$, while VCD traces were recorded with a resolution of $8 \mathrm{~cm}^{-1}$. For hydrocarbon $(+)-\mathbf{1}$, the interferograms of up to 16 individual measurements of 2000 scans each were averaged prior to generation of the infrared spectrum.
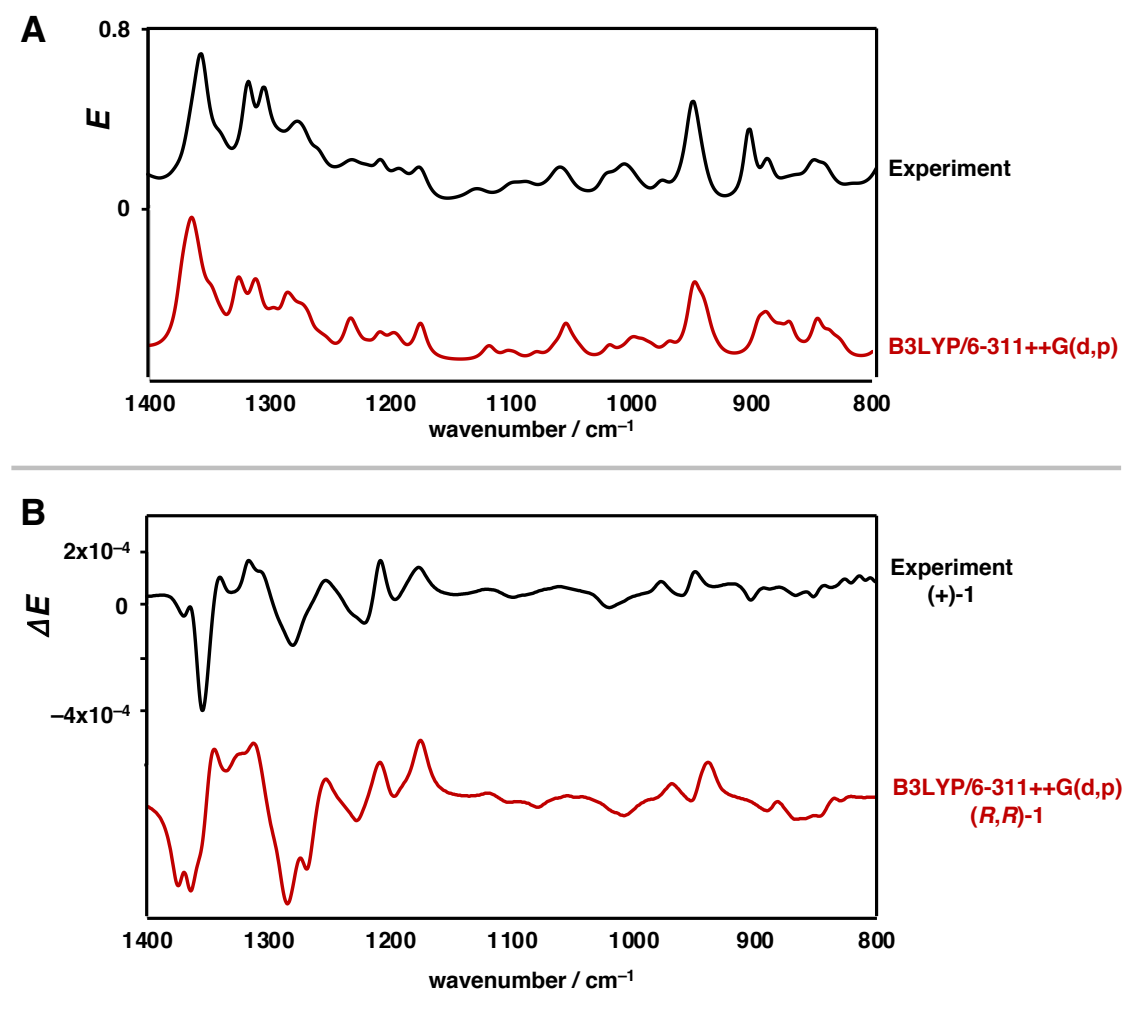

Figure S4. (A) Experimental (neat; black) and computed (B3LYP/6-311++G $(d, p)$ ); gas phase, $6 \mathrm{~cm}^{-1}$ simulated line width, scaled by 0.98 ; red) IR spectra of 1. (B) Experimental (neat; black) and computed (B3LYP/6-311++G $(d, p)$; gas phase, $8 \mathrm{~cm}^{-1}$ line width, scaled by 0.98 ; red) VCD spectra of $(+)-1$ and $(R, R)-1$, respectively. The Boltzmann distribution was calculated based on relative free energies at B3LYP/6$311++\mathrm{G}(d, p)$. 

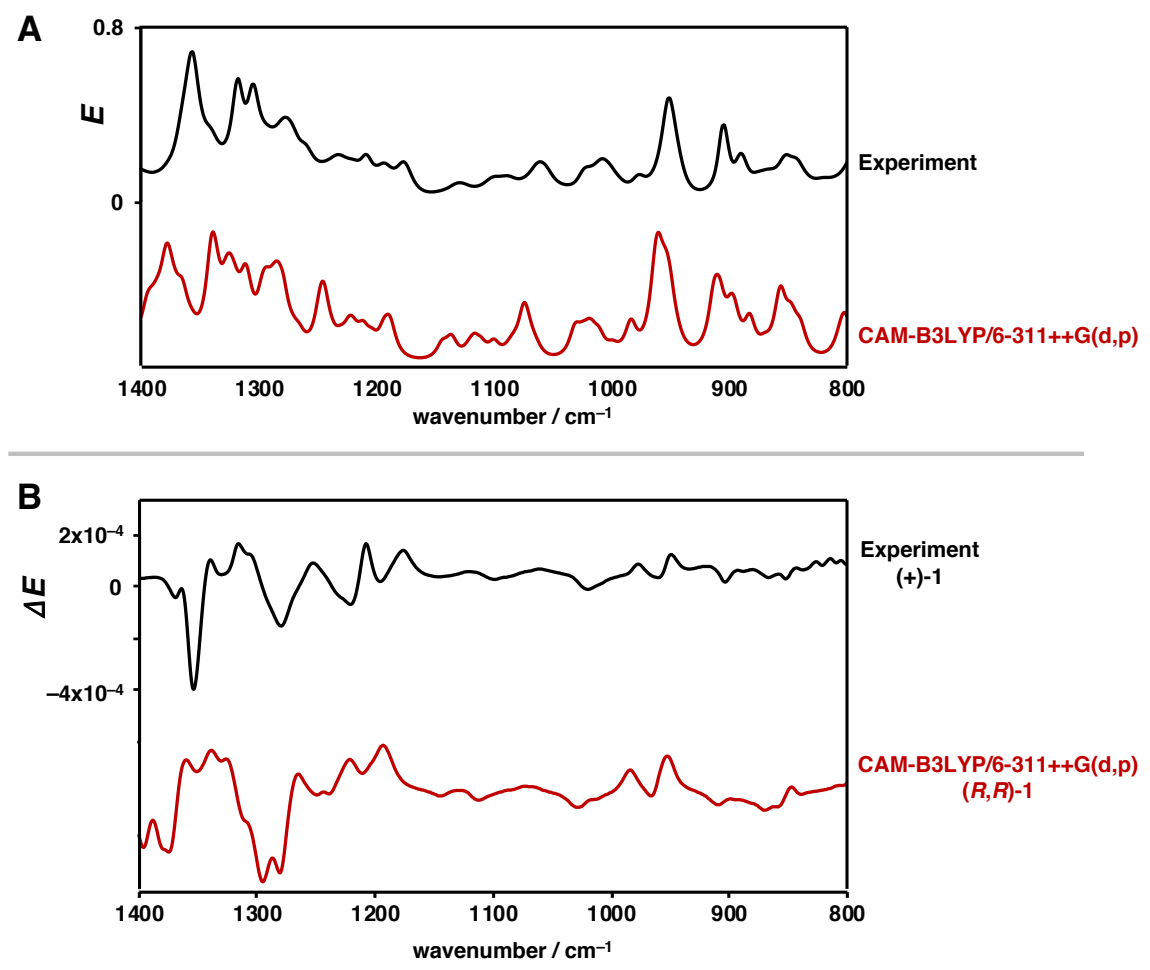

Figure S5. (A) Experimental (neat; black) and computed (CAM-B3LYP/6-311++G(d,p)); gas phase, $6 \mathrm{~cm}^{-1}$ simulated line width, scaled by 0.98 ; red) IR spectra of 1. (B) Experimental (neat; black) and computed (CAMB3LYP/6-311++G $(d, p)$; gas phase, $8 \mathrm{~cm}^{-1}$ line width, scaled by 0.98 ; red) VCD spectra of $(+)-\mathbf{1}$ and $(R, R)-\mathbf{1}$, respectively. The Boltzmann distribution was calculated based on relative free energies at MP2/cc-pVTZ. 


\section{Computational Details}

All computations were performed with the Gaussian16 rev. C program. ${ }^{10}$ For Figure 2, specific rotation for each conformer of 1 was computed at B3LYP/6-311++G(2d,2p) on their MP2/cc-pVTZ optimized structures. The Boltzmann weighted specific rotation was calculated based on the relative free energies (298 K, $1 \mathrm{~atm})$ of the conformers of 1 computed at MP2/cc-pVTZ. For Figure 3, vibrational circular dichroism spectra were computed at $\mathrm{B} 3 \mathrm{LYP} / 6-311++\mathrm{G}(d, p)$ after geometry optimizations of the conformers of $\mathbf{1}$ at the same level of theory. Based on the relative free energies (298 K, $1 \mathrm{~atm})$ of the conformers of 1 computed at MP2/cc-pVTZ, the Boltzmann weighted vibrational circular dichroism spectra were generated using SpecDis $1.71 .^{11}$

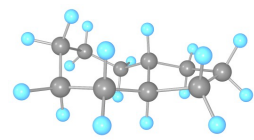

1-I

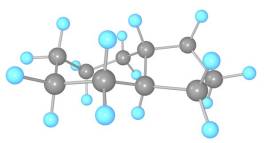

1-II

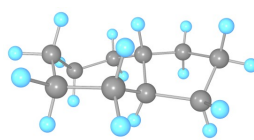

1-III

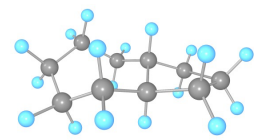

1-IV

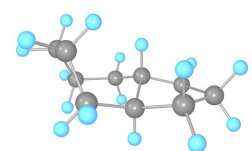

$1-\mathrm{V}$

\section{Cartesian coordinates}

a. MP2/cc-pVTZ

\section{Conformer 1-I}

$$
\begin{array}{r}
-1.852312000 \\
-0.456340000 \\
-0.303176000 \\
0.652448000 \\
0.403289000 \\
0.657245000 \\
2.053613000 \\
2.785909000 \\
2.176020000 \\
2.397212000 \\
1.965481000 \\
3.478849000 \\
1.933806000 \\
2.613207000 \\
1.999307000 \\
0.518707000 \\
0.471257000 \\
0.320521000 \\
-0.590444000 \\
-1.972100000 \\
-0.629334000 \\
-2.818384000 \\
-2.057440000 \\
-1.914884000 \\
-3.198662000 \\
-3.681588000 \\
-2.421300000 \\
-1.861935000
\end{array}
$$

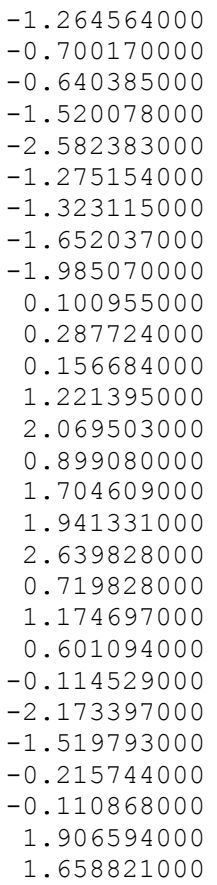

$-0.075823000$

0.194251000

1. 279508000

$-0.454726000$

$-0.394290000$

$-1.520858000$

0.132870000

$-0.607623000$

0.992821000

0.578782000

1. 565153000

0.712765000

$-0.357327000$

$-0.256215000$

$-1.400587000$

$-0.039678000$

1. 028643000

$-0.570189000$

$-0.370557000$

0.119546000

$-1.461392000$

0.252788000

0.489735000

$-1.137301000$

1. 268419000

$-0.410254000$

$-0.550950000$

1. 091859000

\section{Conformer 1-II}


$-1.659912000$

$-1.238234000$

$-0.709055000$

$-1.748999000$

$-2.658416000$

0.626318000

$-0.499167000$

$-1.244119000$

0.197591000

$-1.358482000$

$-1.930654000$

1.718401000

0.428240000

1.020628000

1.256587000

2.534600000

2.134907000

0.289297000

0.387830000

2.133725000

0.851871000

0.017758000

$-1.678302000$

$-2.013853000$

$-0.115865000$

0.351476000

2.055564000

0.920453000

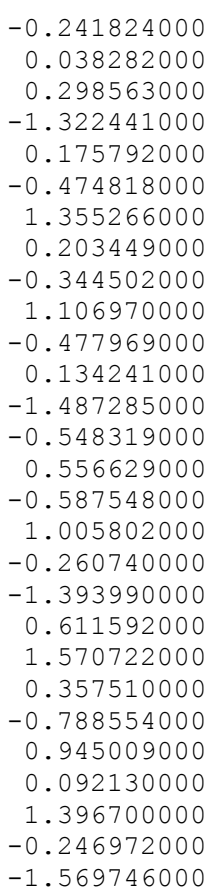

$-0.241824000$

0.038282000

1. 322441000

0.175792000

$-0.474818000$

1.355266000

0.203449000

.344502000

$-0.477969000$

0.134241000

$-1.487285000$

0.548319000

$-0.587548000$

1.005802000

60740000

0.611592000

1.570722000

0.357510000

54000

0.092130000

$-0.246972000$

$-1.569746000$

$$
\begin{gathered}
\boldsymbol{E}=-391.0187458 \text { a.u. } \\
\boldsymbol{Z P V \boldsymbol { E }}=166.70418 \mathrm{kcal} \mathrm{mol}^{-1} \\
\boldsymbol{G}_{\mathbf{2 9 8}}=-390.786818 \text { a.u. }
\end{gathered}
$$

\section{Conformer 1-III}

$\begin{array}{ll}6 & -1.947290000 \\ 6 & -0.463279000 \\ 1 & -0.123431000 \\ 6 & -0.460126000 \\ 6 & -1.907947000 \\ 1 & -0.252459000 \\ 6 & 0.567460000 \\ 1 & 0.635433000 \\ 1 & 0.203679000 \\ 6 & 1.964386000 \\ 1 & 2.012668000 \\ 1 & 2.686276000 \\ 6 & 2.400458000 \\ 1 & 3.490982000 \\ 1 & 2.056110000 \\ 6 & 1.947836000 \\ 1 & 2.262734000 \\ 1 & 2.487604000 \\ 6 & 0.453833000 \\ 1 & 0.274654000 \\ 1 & 0.164611000 \\ 6 & -2.699485000 \\ 1 & -2.105514000 \\ 1 & -2.134678000 \\ 1 & -3.753948000 \\ 1 & -2.638926000 \\ 1 & -2.058052000 \\ 1 & -2.342826000 \\ & \end{array}$

$$
\begin{array}{r}
1.079203000 \\
0.626096000 \\
0.453307000 \\
-0.712311000 \\
-1.198075000 \\
-0.472076000 \\
-1.731598000 \\
-2.524770000 \\
-2.205392000 \\
-1.155001000 \\
-0.758540000 \\
-1.972510000 \\
-0.040345000 \\
-0.031079000 \\
-0.250625000 \\
1.353811000 \\
1.486977000 \\
2.105570000 \\
1.655969000 \\
2.636684000 \\
1.741177000 \\
0.093572000 \\
-1.623678000 \\
-1.961065000 \\
0.002452000 \\
0.413578000 \\
2.113613000 \\
1.018448000
\end{array}
$$

$-0.510731000$ $-0.490012000$ $-1.515958000$

0.280363000

0.215583000

1.329071000

$-0.191288000$

0.557592000

$-1.107293000$

$-0.450079000$

$-1.467023000$

$-0.413948000$

0.507499000

0.553096000

1. 523687000

0.063806000

$-0.975599000$

0.644062000

0.163925000

$-0.284557000$

1. 216873000

0.392804000

$-0.772878000$

0.960754000

0.135810000

1. 434983000

$-0.184719000$

$-1.525184000$

$$
\begin{gathered}
\boldsymbol{E}=-391.0183625 \text { a.u. } \\
\boldsymbol{Z P V E}=166.67171 \mathrm{kcal} \mathrm{mol}^{-1} \\
\boldsymbol{G}_{\mathbf{2 9 8}}=-390.786634 \text { a.u. }
\end{gathered}
$$

\section{Conformer 1-IV}


b. B3LYP/6-311++G(d,p)

\section{Conformer 1-I}

$\begin{array}{rr}-1.882522000 & -1.274575000 \\ -0.470692000 & -0.707421000 \\ -0.305537000 & -0.643900000 \\ 0.640989000 & -1.547440000 \\ 0.396621000 & -2.611059000 \\ 0.625222000 & -1.346777000 \\ 2.069298000 & -1.336552000 \\ 2.775294000 & -1.678603000 \\ 2.224506000 & -1.998394000 \\ 2.455614000 & 0.091865000 \\ 2.095820000 & 0.270844000 \\ 3.548319000 & 0.132454000 \\ 1.955931000 & 1.247288000 \\ 2.625888000 & 2.103287000 \\ 2.029422000 & 0.974600000 \\ 0.527182000 & 1.709206000 \\ 0.479733000 & 1.932436000 \\ 0.327801000 & 2.657968000 \\ -0.591973000 & 0.726287000 \\ -1.990977000 & 1.190162000 \\ -0.611764000 & 0.618786000 \\ -2.854107000 & -0.097837000 \\ -2.095531000 & -2.147150000 \\ -1.954927000 & -1.604418000 \\ -3.298771000 & -0.170670000 \\ -3.683141000 & -0.102471000 \\ -2.423261000 & 1.924116000 \\ -1.908152000 & 1.683046000\end{array}$

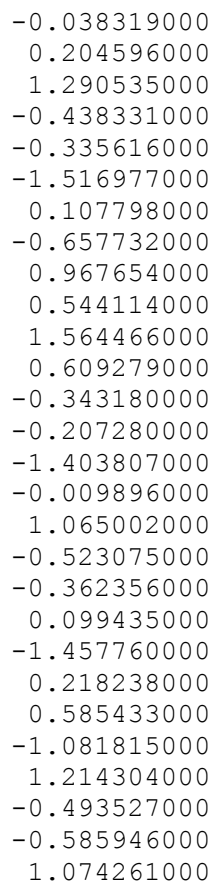

\section{Conformer 1-II}

\section{Conformer 1-III}




$\begin{array}{rr}0.614947000 & 2.528239000 \\ 0.212834000 & 2.227403000 \\ 1.987020000 & 1.192917000 \\ 2.063409000 & 0.851672000 \\ 2.689952000 & 2.028415000 \\ 2.456348000 & 0.040058000 \\ 3.551862000 & 0.032924000 \\ 2.154238000 & 0.221067000 \\ 1.986121000 & -1.352205000 \\ 2.271184000 & -1.472087000 \\ 2.548462000 & -2.115311000 \\ 0.489627000 & -1.667145000 \\ 0.308017000 & -2.654541000 \\ 0.240426000 & -1.762783000 \\ -2.767801000 & -0.066252000 \\ -2.097660000 & 1.643219000 \\ -2.179634000 & 1.986028000 \\ -3.793174000 & 0.036773000 \\ -2.829917000 & -0.329842000 \\ -2.057858000 & -2.124803000 \\ -2.341443000 & -1.233159000\end{array}$

\section{Conformer 1-IV}

$\begin{array}{rr}-0.350460000 & 2.616352000 \\ 0.000000000 & 1.603368000 \\ 1.087975000 & 1.680857000 \\ -0.674229000 & 1.144898000 \\ -0.765463000 & 1.999726000 \\ -1.703736000 & 0.856811000 \\ 0.000000000 & 0.000000000 \\ -0.759791000 & -0.428036000 \\ 0.759791000 & 0.428036000 \\ 0.674229000 & -1.144898000 \\ 1.703736000 & -0.856811000 \\ 0.765463000 & -1.999726000 \\ 0.000000000 & -1.603368000 \\ 0.350460000 & -2.616352000 \\ -1.087975000 & -1.680857000 \\ 0.329508000 & -0.695582000 \\ 1.419193000 & -0.534252000 \\ -0.048079000 & -1.238101000 \\ -0.329508000 & 0.695582000 \\ 0.048079000 & 1.238101000 \\ -1.419193000 & 0.534252000 \\ 0.000000000 & 0.000000000 \\ -1.061803000 & -1.653589000 \\ 0.614698000 & -2.043617000 \\ -0.875341000 & 0.030524000 \\ 0.875341000 & -0.030524000 \\ 1.061803000 & 1.653589000 \\ -0.614698000 & 2.043617000\end{array}$

$-0.388287000$ $-0.619106000$ $-0.746114000$ $-1.925645000$ $-2.604426000$ $-1.681618000$ $-2.714931000$ $-3.379031000$ $-3.379031000$ $-1.925645000$ $-1.681618000$ $-2.604426000$ $-0.619106000$ $-0.388287000$ $-0.746114000$ 0.570278000 0.562171000 1. 960197000 0.570278000 1.960197000 0.562171000 2.900150000 1. 925906000 2. 287782000 3.553616000 3. 553616000 1.925906000 2. 287782000

\section{Conformer 1-V}


1.230562000

0.373595000

$-0.022484000$

1.833410000

1.883287000

0.079548000

$-0.161132000$

$-1.775151000$

$-1.932266000$
$-0.240484000$

$-1.319796000$

$-0.186165000$

0.665422000

$-1.088194000$

0.604091000

$-1.119669000$

$-0.840578000$ 


\section{References}

[1] Ayres, D. C.; Raphael, R. A. J. Chem. Soc. 1958, 1779-1789.

[2] Sicher, J.; Šipoš, F.; Jonáš, J. Collect. Czech. Chem. Commun. 1961, 26, 262-275.

[3] Saito, F.; Becker, J.; Schreiner, P. R. J. Org. Chem. 2020, 85, 4441-4447.

[4] Krause, L.; Herbst-Irmer, R.; Sheldrick, G. M.; Stalke, D. J. Appl. Cryst. 2015, 48, 3.

[5] Sheldrick, G. M. Acta Cryst. A 2015, 71, 3.

[6] Sheldrick, G. M. Acta Cryst. C 2015, 71, 3.

[7] Müller, P. Cryst. Rev. 2009, 15(1), 57.

[8] Parsons, S.; Flack, H. D.; Wagner, T. Acta Cryst. B 2013, 69, 249.

[9] https://www.ccdc.cam.ac.uk/structures/

[10] Gaussian 16, Revision C.01, Frisch, M. J.; Trucks, G. W.; Schlegel, H. B.; Scuseria, G. E.; Robb, M. A.; Cheeseman, J. R.; Scalmani, G.; Barone, V.; Petersson, G. A.; Nakatsuji, H.; Li, X.; Caricato, M.; Marenich, A. V.; Bloino, J.; Janesko, B. G.; Gomperts, R.; Mennucci, B.; Hratchian, H. P.; Ortiz, J. V.; Izmaylov, A. F.; Sonnenberg, J. L.; Williams-Young, D.; Ding, F.; Lipparini, F.; Egidi, F.; Goings, J.; Peng, B.; Petrone, A.; Henderson, T.; Ranasinghe, D.; Zakrzewski, V. G.; Gao, J.; Rega, N.; Zheng, G.; Liang, W.; Hada, M.; Ehara, M.; Toyota, K.; Fukuda, R.; Hasegawa, J.; Ishida, M.; Nakajima, T.; Honda, Y.; Kitao, O.; Nakai, H.; Vreven, T.; Throssell, K.; Montgomery, J. A., Jr.; Peralta, J. E.; Ogliaro, F.; Bearpark, M. J.; Heyd, J. J.; Brothers, E. N.; Kudin, K. N.; Staroverov, V. N.; Keith, T. A.; Kobayashi, R.; Normand, J.; Raghavachari, K.; Rendell, A. P.; Burant, J. C.; Iyengar, S. S.; Tomasi, J.; Cossi, M.; Millam, J. M.; Klene, M.; Adamo, C.; Cammi, R.; Ochterski, J. W.; Martin, R. L.; Morokuma, K.; Farkas, O.; Foresman, J. B.; Fox, D. J. Gaussian, Inc., Wallingford CT, 2016.

[11] Bruhn, T.; Schaumlöffel, A.; Hemberger, Y.; Pescitelli, G. SpecDis version 1.71, Berlin, Germany, 2017, http:/specdis-software.jimdo.com. 